\title{
Corticobulbar projections from distinct motor cortical areas to the reticular formation in macaque monkeys
}

\author{
Michela Fregosi, Alessandro Contestabile, ${ }^{*}$ Adjia Hamadjida ${ }^{\dagger}$ and Eric M. Rouiller (iD \\ Department of Medecine, University of Fribourg, Chemin du Musée 5, 1700 Fribourg, Switzerland
}

Keywords: anterograde tracing, brainstem, motor cortex, non-human primate, premotor cortex

\begin{abstract}
Corticospinal and corticobulbar descending pathways act in parallel with brainstem systems, such as the reticulospinal tract, to ensure the control of voluntary movements via direct or indirect influences onto spinal motoneurons. The aim of this study was to investigate the corticobulbar projections from distinct motor cortical areas onto different nuclei of the reticular formation. Seven adult macaque monkeys were analysed for the location of corticobulbar axonal boutons, and one monkey for reticulospinal neurons' location. The anterograde tracer BDA was injected in the premotor cortex (PM), in the primary motor cortex (M1) or in the supplementary motor area (SMA), in 3, 3 and 1 monkeys respectively. BDA anterograde labelling of corticobulbar axons were analysed on brainstem histological sections and overlapped with adjacent Nissl-stained sections for cytoarchitecture. One adult monkey was analysed for retrograde CB tracer injected in C5-C8 hemispinal cord to visualise reticulospinal neurons. The corticobulbar axons formed bilateral terminal fields with boutons terminaux and en passant, which were quantified in various nuclei belonging to the Ponto-Medullary Reticular Formation (PMRF). The corticobulbar projections from both PM and SMA tended to end mainly ipsilaterally in PMRF, but contralaterally when originating from M1. Furthermore, the corticobulbar projection was less dense when originating from M1 than from non-primary motor areas (PM, SMA). The main nuclei of bouton terminals corresponded to the regions where reticulospinal neurons were located with $C B$ retrograde tracing. In conclusion, the corticobulbar projection differs according to the motor cortical area of origin in density and laterality.
\end{abstract}

\section{Introduction}

The corticobulbar and corticospinal projections act in parallel with brainstem descending pathways to ensure both direct and indirect control on motoneurons of the spinal cord. The corticobulbar projections establish connections from the cerebral cortex to brainstem nuclei, some of them giving rise to descending pathways towards the spinal cord, such as the reticulospinal tract (RST) (Kuypers, 1981; Lemon, 2008). The RST originates from the Ponto-Medullary Reticular Formation (PMRF) (e.g. Kuypers, 1981; Matsuyama \& Drew, 1997; Matsuyama et al., 1997, 1999; Sakai et al., 2009) and sends bilateral projections to the intermediate zone of the spinal cord (Lawrence \& Kuypers, 1968a,b; Kuypers, 1981; Lemon, 2008). The PMRF is composed of several nuclei some of them contributing to the RST: the Gigantocellular reticular nucleus (Gi) and the Pontine

Correspondence: Prof. E. M. Rouiller, as above.

E-mail: Eric.Rouiller@unifr.ch

The associated peer review process communications can be found in the online version of this article.

* Present address: Department of Fundamental Neurosciences, University of Geneva, Rue Michel-Servet 1, 1211, Geneva 4, Switzerland

${ }^{\dagger}$ Present address: Department of Neuroscience, Centre de Recherche du Centre Hospitalier de l'Université de Montréal, Montreal (CRCHUM), QC, Canada reticular nucleus Pars caudalis $(\mathrm{PnC})$ and Pars oralis $(\mathrm{PnO})$ (Kuypers, 1981; Sakai et al., 2009). The RST system has been demonstrated to be involved in the control of posture and locomotion (Lawrence \& Kuypers, 1968a,b; Drew et al., 1986; Matsuyama \& Drew, 1997; Matsuyama et al., 1999, 2004; Schepens \& Drew, 2004, 2006; Schepens et al., 2008; Lemon et al., 2012), as well as of postural adjustments during reaching movements (Schepens \& Drew, 2004, 2006; Schepens et al., 2008). The role of the RST in reaching movements has been demonstrated in monkeys (Buford \& Davidson, 2004; Davidson \& Buford, 2004, 2006; Davidson et al., 2007). Electrophysiological studies conducted on the medial PMRF (mPMRF) in macaques showed neuronal preparatory activity, suggesting that mPMRF is also involved in movement preparation (Buford \& Davidson, 2004). Recent studies have demonstrated that the RST sends monosynaptic or disynaptic projections to motoneurons controlling intrinsic hand muscles (Riddle et al., 2009; Riddle \& Baker, 2010; Soteropoulos et al., 2012). Moreover, spinal interneurons receive convergent information from the RST and the corticospinal tract (CST) (Ortiz-Rosario et al., 2014), the latter being the main player in the control of independent finger movements (e.g. Lawrence \& Kuypers, 1968a,b; Lemon, 1993, 2008, 2010; Lemon \& Griffiths, 2005; Schieber, 2007; Riddle \& Baker, 2010). Altogether these results, including the role of the RST in the control 
of distal hand muscles in humans (Honeycutt et al., 2013), suggest that the RST system is more involved in hand control than it was previously believed. Furthermore, the RST system has been shown in monkeys to facilitate forearm flexor muscles and intrinsic hand muscles after a lesion of the CST, which was not the case of the forearm extensors (Zaaimi et al., 2012).

Several studies have been conducted on rodents PMRF to understand its function and anatomy (e.g. Valverde, 1961, 1962; Esposito et al., 2014; Alstermark \& Ekerot, 2015; Liang et al., 2015, 2016) as well as its contribution after spinal cord or stroke lesions (Bachmann et al., 2014; Zörner et al., 2014; García-Alías et al., 2015). In contrast, current knowledge on the corticobulbar projections in monkeys is limited, both anatomically and functionally. An early study of the corticobulbar projections to the reticular formation was performed by Kuypers (1958), based on the anterograde degeneration method, extended later in a subsequent report (Kuypers \& Lawrence, 1967). The authors observed that the lateral tegmentum of the brainstem receives bilateral inputs from the lower one-third of the pericentral areas along the central sulcus, whereas the middle one-third of the precentral gyrus and rostral premotor cortex (PM) send projections to the medial tegmental field of the upper Medulla oblongata (Kuypers, 1958). More recently, retrograde tracer studies on macaque monkeys have demonstrated that the CST gives rise to collaterals contacting the medial reticular formation, corresponding to cortical control on mPMRF (Keizer \& Kuypers, 1989). Moreover, a recent study conducted by Borra et al. (2010) has shown that the ventral PM (PMv) hand field sends a projection to the PMRF.

The main aim of this study was to use modern tracing techniques with cellular resolution in order to trace the corticobulbar projections in intact adult macaque monkeys from several motor cortical areas terminating in the reticular formation, thus possibly influencing in turn the RST. To this end we analysed the corticobulbar projections from the primary motor cortex (M1), the premotor cortex (PM) and the supplementary motor area (SMA), focusing on the PMRF. Moreover, we compared the spatial distribution of corticobulbar terminals with the location of reticulospinal neurons in the PMRF after injection of retrograde tracer in the cervical spinal cord (C5-C8). We thereby tested the hypothesis that corticobulbar projections arising from distinct motor cortical areas differ with respect to their laterality, spatial distribution, and density along the rostro-caudal axis, as well as to the targeted PMRF nuclei.

\section{Materials and Methods}

The corticobulbar projections arising from distinct motor cortical areas were investigated in seven adult macaque monkeys (Macaca fascicularis; two females and five males, aged 4-10 years, weighing between 4-10 kg; see Table 1). In addition, one monkey (Macaca fascicularis, 4 years old, $4 \mathrm{~kg}$; see Table 1) was used to identify the origin of the reticulospinal tract in the PMRF. The animals were not involved in any behavioural protocol. Animal care, surgical procedures and experiments were all conducted in accordance to the Guide and Care and Use of Laboratory Animals (ISBN 0-30905377-3; 1996) as well as authorised by local (canton of Fribourg) and federal (Swiss) veterinary offices (veterinary authorizations $\mathrm{N}^{\circ}$ 156-08E, 156-02, 44-92-3). The number of monkeys was minimised, by limiting the number of cases per motor cortical area and by using the same animal to trace other pathways as well (e.g. Rouiller et al., 1994, 1996; Hamadjida et al., 2012). The authors adhere to the NC3Rs initiative and the Basel declaration (www.basel-declaration. org).

\section{Surgical procedures and tracer injections}

The monkeys were deeply anaesthetised according to protocols previously published (Rouiller et al., 1994, 1996; Hamadjida et al., 2012; Wyss et al., 2013), aimed to minimise pain and discomfort. Briefly, the monkeys were sedated with ketamine $(5 \mathrm{mg} / \mathrm{kg}$ body weight, i.m.) and pre-medicated, in particular with the analgesic carprofen (Rymadil, $4 \mathrm{mg} / \mathrm{kg}$, s.c.) in order to reduce pain immediately after surgery. Under aseptic conditions, the surgery was conducted under deep anaesthesia obtained by i.v. infusion of propofol (mixture of $1 \%$ propofol and $4 \%$ glucose in saline, 1 volume of propofol and 2 volumes of glucose delivered at the rate of $0.1 \mathrm{ml} /$ $\mathrm{min} / \mathrm{kg}$ ). To reduce pain, ketamine was added to the perfusion solution to be delivered at a rate of $0.0625 \mathrm{mg} / \mathrm{min} / \mathrm{kg}$. After surgery, the animals were treated daily with antibiotics (Ampiciline 30\%, $30 \mathrm{mg} / \mathrm{kg}$, s.c.) and analgesics (pills of Rymadil in food) for a period of 7-10 days.

Seven animals used for identifying corticobulbar projections were injected using a $10 \mu \mathrm{l}$ Hamilton micro-syringe unilaterally with the anterograde tracer biotinylated dextran amine (BDA; MW = 10 000; Molecular Probe, Eugene, OR, USA). BDA concentrations were 5\% (in distilled water) for M1-3, SMA-1 and 10\% for PM-1, PM-2, PM-3, M1-1 and M1-2 (see Table 1 for injection sites). For both M1-3 and SMA-1 the hand area was identified with intracortical microstimulation (ICMS; see Rouiller et al., 1994, 1996) whereas, for the other animals ICMS was not performed and therefore the injections were not restricted to the hand region. In SC-1 the retrograde tracer cholera toxin B subunit (CB; List Biological Laboratories, Campbell, Calif, USA; $0.05 \%$ solution in distilled water) was injected unilaterally under deep anaesthesia in the cervical spinal cord at multiple sites between C5 and C8 (Rouiller et al., 1994).

\section{Histology}

After completion of tracer injections and a survival period of 2029 days for BDA, around 5 days for $\mathrm{CB}$, to allow transport of the tracers to the region of interest, the animals were euthanized under deep anaesthesia (lethal dose of pentobarbital sodium $90 \mathrm{mg} / \mathrm{kg}$ body weight; i.p.) and perfused transcardially as previously reported (Rouiller et al., 1994, 1996; Hamadjida et al., 2012; Wyss et al., 2013). The brainstem was cut in the frontal plane in $50 \mu \mathrm{m}(40 \mu \mathrm{m}$ for SC-1 only) thick sections with a freezing microtome, collected in 5-7 series (see Table 1). In the animals injected in motor cortical areas, one series of brainstem sections was processed to visualise BDA in order to reconstruct the location of stem axons and axonal boutons, both en passant and terminaux (histochemical protocol described in Rouiller et al., 1994). In the animal injected in the cervical cord, one series of sections was processed to visualise $\mathrm{CB}$ in order to identify the reticulospinal neurons in the PMRF (immunohistochemical protocol described in Rouiller et al., 1994). The adjacent corresponding Nissl stained sections from a second series were used to identify and delineate the brainstem nuclei (Table 2).

\section{Data acquisition}

BDA and $\mathrm{CB}$ stained sections were first observed with a light microscope, Olympus BH-2 (Olympus Schweiz SA) to determine the rostro-caudal extent of the brainstem to analyse. BDA sections were taken caudally from above the Decussatio pyramidis $(-11.85 \mathrm{~mm}$ from the interaural zero coordinate, Paxinos et al., 2000) to rostrally at the beginning of the Pontine nuclei (the most rostral section analysed was the one on which there was dense BDA 
TABLE 1. Summary of the individual data for each of the eight monkeys involved in this study

\begin{tabular}{|c|c|c|c|c|c|c|c|c|}
\hline & $\begin{array}{l}\text { PM-1 } \\
\text { Mk-R13 }\end{array}$ & $\begin{array}{l}\text { PM-2 } \\
\text { Mk-R12 }\end{array}$ & $\begin{array}{l}\text { PM-3 } \\
\text { Mk-CH }\end{array}$ & $\begin{array}{l}\text { M1-1 } \\
\text { Mk-Z182 }\end{array}$ & $\begin{array}{l}\text { M1-2 } \\
\text { Mk-M310 }\end{array}$ & $\begin{array}{l}\text { M1-3 } \\
\text { Mk-M93-80 }\end{array}$ & $\begin{array}{l}\text { SMA-1 } \\
\text { Mk-M93-81 }\end{array}$ & $\begin{array}{l}\text { SC-1 } \\
\text { Mk-90-60 }\end{array}$ \\
\hline Sex & Female & Female & Male & Male & Male & Male & Male & Male \\
\hline Age & 4.5 & 6 & 10 & 7.5 & 8.5 & 4 & 4 & 4 \\
\hline Weight & 4 & 4 & 6 & 10 & 10 & 4 & 4 & 4 \\
\hline Tracer injected & BDA & BDA & BDA & BDA & BDA & BDA & BDA & $\mathrm{CB}$ \\
\hline Number of brainstem's sections series & 5 & 5 & 5 & 6 & 6 & 7 & 6 & 6 \\
\hline Distance between sections & $250 \mu \mathrm{m}$ & $250 \mu \mathrm{m}$ & $250 \mu \mathrm{m}$ & $300 \mu \mathrm{m}$ & $300 \mu \mathrm{m}$ & $350 \mu \mathrm{m}$ & $300 \mu \mathrm{m}$ & $240 \mu \mathrm{m}$ \\
\hline Interval between the analysed sections & 1 out 4 & 1 out 4 & 1 out 4 & 1 out 3 & 1 out 3 & 1 out 3 & 1 out 3 & 1 out 4 \\
\hline Distance between the analysed sections & $1 \mathrm{~mm}$ & $1 \mathrm{~mm}$ & $1 \mathrm{~mm}$ & $0.9 \mathrm{~mm}$ & $0.9 \mathrm{~mm}$ & $1.05 \mathrm{~mm}$ & $0.9 \mathrm{~mm}$ & $0.96 \mathrm{~mm}$ \\
\hline Injected side & Left & Left & Left & Right & Right & Right & Right & Right \\
\hline Injection sites & $\mathrm{PMd} / \mathrm{PMv}$ & PMd & $\mathrm{PMd} / \mathrm{PMv}$ & M1 & M1 & M1 & SMA & $\mathrm{C} 5-\mathrm{C} 8$ \\
\hline Volume of tracer injected $(\mu \mathrm{l})$ & 8.8 & 7.2 & 8 & 25.5 & 22.5 & 10.5 & 9 & 4 \\
\hline No. of sites injected per tracer & 11 & 9 & 10 & 17 & 15 & 7 & 6 & 3 \\
\hline Number of BDA labelled CS axons & 1802 & 1473 & 1201 & 950 & 703 & 3195 & 2160 & - \\
\hline
\end{tabular}

In $\mathrm{SC}-1$, the tracer $\mathrm{CB}$ was injected in the cervical cord (C5-C8). The weight (rounded to the $\mathrm{kg}$ ) was determined at the time of euthanasia, as well as the age (rounded to 0.5 year). CS, Corticospinal.

TABLE 2. Abbreviations for the nuclei of the brainstem delineated in this study

\begin{tabular}{|c|c|c|c|}
\hline $6 \mathrm{~N}$ & Abducens nucleus & mcp & Middle cerebellar peduncle \\
\hline $7 \mathrm{~N}$ & Facial nucleus & $\mathrm{ml}$ & $\begin{array}{l}\text { Medial lemniscus (sometimes } \\
\text { including the trapezial body) }\end{array}$ \\
\hline $12 \mathrm{~N}$ & Hypoglossal nucleus & Mo5 & Trigeminal motor nucleus \\
\hline $5 n$ & Trigeminal nerve & $\mathrm{PnC}$ & Pontine reticular nucleus caudal \\
\hline $7 n$ & Facial nerve & $\mathrm{PnO}$ & Pontine reticular nucleus oral \\
\hline $8 n$ & $\begin{array}{l}\text { Vestibulocochlear } \\
\text { nerve }\end{array}$ & $\begin{array}{l}\text { Pont } \\
\text { Nucl }\end{array}$ & Pontine nuclei \\
\hline $9 n$ & $\begin{array}{l}\text { Glossopharyngeal } \\
\text { nerve }\end{array}$ & $\operatorname{Pr}$ & Prepositus nucleus \\
\hline $\mathrm{CN}$ & Cochlear nucleus & Pr5 & $\begin{array}{l}\text { Principal sensory nucleus } \\
\text { trigeminal nerve }\end{array}$ \\
\hline $\mathrm{CU}$ & Cuneate nucleus & Py & Pyramidal tract \\
\hline $\mathrm{ECU}$ & $\begin{array}{l}\text { External cuneate } \\
\text { nucleus }\end{array}$ & Raphe & Raphe nuclei \\
\hline DTg & $\begin{array}{l}\text { Dorsal tegmental } \\
\text { nucleus }\end{array}$ & RPO & Rostral preolivary compley \\
\hline Gi & $\begin{array}{l}\text { Gigantocellular } \\
\text { reticular nucleus }\end{array}$ & RtTg & Reticulo tegmental nucleus of Pons \\
\hline $\mathrm{IO}$ & Inferior olive & $\mathrm{SOC}$ & Superior olivary complex \\
\hline IRt & $\begin{array}{l}\text { Intermediate reticular } \\
\text { nucleus }\end{array}$ & Sol & Solitary nucleus \\
\hline 11 & Lateral lemniscus & Sp5 & Spinal sensory trigeminal nucleus \\
\hline $\mathrm{LC}$ & Locus coeruleus & VC & Vestibular complex \\
\hline LRt & $\begin{array}{l}\text { Lateral reticular } \\
\text { nucleus }\end{array}$ & & \\
\hline
\end{tabular}

staining in the most caudal part of Pontine nuclei; $+00.75 \mathrm{~mm}$ from the interaural zero coordinate). For CB stained sections the analysed rostro-caudal extent was $-12.75 \mathrm{~mm}$ to $+02.55 \mathrm{~mm}$. This relevant portion of the brainstem included usually 12 sections from an individual series of sections (15 for SC-1), which were scanned using an Olympus BX40 microscope interfaced with the Neurolucida ${ }^{\circledR}$ software (MBF, Bioscience-MicroBrightField, Inc. Version 9 for PM-1, PM-2, PM-3, M1-1, M1-2 and Version 11 for SMA-1; M1-3, SC-1). In general, the interval between consecutive analysed sections ranged between $1 \mathrm{~mm}$ (PM-1, PM-2, PM-3 and M1-3) and $0.9 \mathrm{~mm}$ (M1-1, M1-2 and SMA-1), and $0.96 \mathrm{~mm}$ for SC-1 (Table 1). Intervals between sections were maintained constant, with the exception of sections 4 and 5 of PM- 3 separated by $0.75 \mathrm{~mm}$ (instead of $1 \mathrm{~mm}$ ). In order to detect a possible anteroposterior difference in the corticobulbar projection, the brainstem was subdivided into a rostral half (sections 1-6) and in a caudal half (sections $7-$ $12)$, as a constant number of analysed sections $(n=12)$ usually covered the zone of interest (PMRF) in each animal.

\section{Data analysis of $B D A, C B$ and Niss/ stained sections}

BDA stained sections comprising the PMRF were first scanned with Neurolucida ${ }^{\circledR}$ at $40 \times$ and contours of both the section and Pyramidal tracts were drawn. A midline was drawn in order to separate the ipsilateral side from the contralateral side with respect to the BDA injected hemisphere. Afterwards, the same sections were systematically scanned at $100 \times$ in order to plot visible stem axons (Fig. 3A) except those located in the Pyramids. Finally, the sections were scanned a third time at $200 \times$ and axonal boutons en passant and terminaux were plotted (Fig. 3B and C). To further subdivide the ipsilateral side from the contralateral side different colours were used to plot stem axons and boutons. To be considered as bouton en passant or terminal, an axonal bouton along an axon segment, visible at magnification $200 \times$, should exhibit a diameter at least twice that of the axon's diameter. In BDA stained sections Pontine nucle $i$ were drawn when visible, but they were not scanned as it is known that Pontine nuclei receive very dense corticofugal axon terminals (Wiesendanger et al., 1979; Borra et al., 2010). As compared to other corticofugal projections (e.g. corticostriatal, corticothalamic, corticorubral, corticospinal) which gave rise to highly dense axonal terminal fields, the present corticobulbar terminal fields labelled with BDA were much less dense, allowing charting most stem axons and all boutons present in PMRF on the analysed histological sections.

$\mathrm{CB}$ stained sections comprising the PMRF were scanned with Neurolucida ${ }^{\circledR}$ software at $40 \mathrm{x}$, and contours of the section were drawn. A midline was drawn in order to separate the ipsilateral side from the contralateral side with respect to the $\mathrm{CB}$ injected hemispinal cord. Afterwards, the same sections were scanned at $100 \times$ in order to plot visible CB stained neurons (Fig. 3D). To further subdivide the ipsilateral side from the contralateral side different colours and symbols were used to plot the CB stained neurons.

Photomicrographs of Nissl stained sections adjacent to the analysed BDA/CB stained sections were captured at $12.5 \times$. Afterwards, based on Paxinos et al. (2000), brainstem nuclei were identified on the photomicrographs and both section and nuclei contours were drawn on the corresponding Nissl stained sections (Fig. 1). The identified nuclei are summarised in Table 2. 


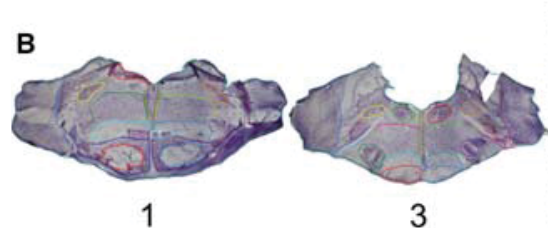

1 3
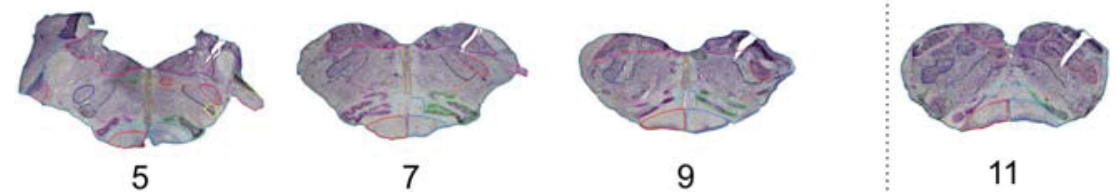

11
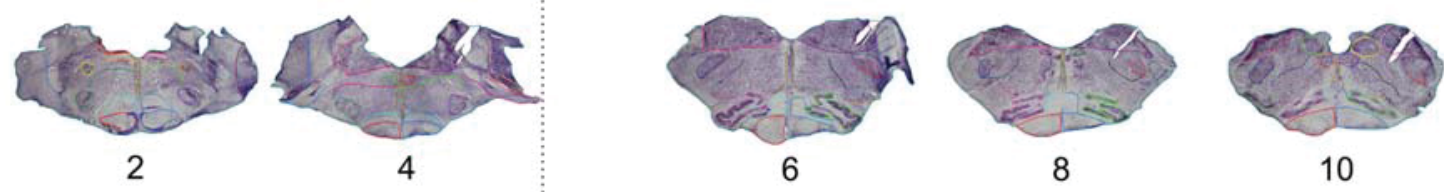

10

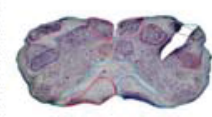

12

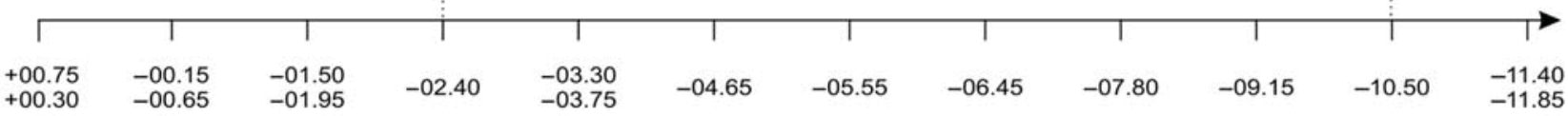

(1)

(2)

(3)

(4)

(5)

(6)

(7)

(8)

(9)

(10)

(11)

(12)

\section{Rostral}

FIG. 1. Rostro-Caudal extent of the analysed brainstem. (A) Graphical representation of the location of the brainstem nuclei or group of nuclei according to their rostral to caudal position and extent. (B) Photomicrographs of 12 coronal brainstem Nissl-stained sections overlapped with the delineation of the nuclei of interest, covering the analysed brainstem rostrocaudal extent. Along the abscissa, the corresponding rostrocaudal coordinates (or range of coordinates) in the Paxinos et al. (2000) atlas are indicated above each section number given between parentheses. *Comprises the following nuclei: Pontine nuclei, Mo5, Pr5, Sp5 $6 \mathrm{~N}, 7 \mathrm{~N}, 12 \mathrm{~N}$, SOC, IO RtTg, VC, CU, ECU, Pr and Sol nuclei (see Table 2 for the abbreviation list).

\section{Sections superposition, analysis and statistics}

Files obtained using the Neurolucida ${ }^{\circledR}$ software for BDA, CB and Nissl were saved in.DAT format. These files were then converted in PDF format and imported in CorelDRAW X7. BDA and CB sections were superimposed onto Nissl stained sections and adjusted to obtain the best alignment. For SC-1 (CB injection) contours were all derived from Nissl-stained sections, whereas for BDA injected animals contours were all derived from Nissl-stained sections with the only exception of the pyramidal tracts obtained from the BDAstained sections.

Superimposed sections were used to quantify the number of boutons among the brainstem nuclei. For each monkey injected with BDA, we established the number of labelled boutons on both the ipsilateral and contralateral sides for each individual section, as well as the total number of boutons for the whole brainstem extent for both the ipsilateral and the contralateral halves of the brainstem and calculated the corresponding percentages (global percentages; Table 3). Data of BDA sections were then normalised according to the total number of BDA labelled corticospinal axons, as assessed from a section just above the Decussatio pyramidis.

Moreover, for SC-1 we established the number of labelled reticulospinal neurons in each individual section for the ipsilateral and the contralateral halves of the PMRF, as well as the percentages of reticulospinal neurons found in each nucleus of the PMRF for both the ipsilateral and contralateral sides (Table 4). Global percentages were calculated for SC-1 as well (Table 3). For all animals the distribution of either the axonal boutons (en passant and terminaux; $\mathrm{BDA}$ injection) or the reticulospinal neurons ( $\mathrm{CB}$ injection) along the rostro-caudal axis were plotted (Fig. 7).
Statistics using parametric paired $t$-test (and non-parametric Wilcoxon when required) were used to compare on each section the ipsilateral side with the contralateral side of the brainstem for animals injected with BDA. Specifically we compared: (i) the total number of boutons on both ipsilateral and contralateral sides, (ii) the total number of boutons on the ipsilateral and contralateral caudal half of the brainstem (sections 7-12); (iii) the total number of boutons on the ipsilateral and contralateral rostral half of the brainstem (sections 16); (iv) the number of ipsilateral boutons and the number of contralateral boutons for each nucleus or group of nuclei. For the latter, statistics were calculated on the basis of the number of boutons in each nucleus and were derived from the paired $t$-test/Wilcoxon, represented with asterisks: $* P \leq 0.05 ;{ }^{* *} P \leq 0.01,{ }^{* * *} P \leq 0.001$.

As recommended in previous tracing reports (e.g. Geuna, 2000; Lavenex et al., 2000; Benes \& Lange, 2001), stereology was not used to reconstruct the distribution of BDA labelled axonal boutons in PMRF. Stereology is most adequate when the number of elements of interest is too large to be pointed individually and/or when attempting to determine their total absolute number in a structure. It was not the goal in this study to establish the total number of corticobulbar boutons, but rather to compare their relative density between cases based on similar sampling of analysed sections and normalisation procedures. Furthermore, in each individual section, the number of corticobulbar boutons was relatively low so that all of them were exhaustively pointed, within a spatially limited territory (brainstem in the present case).

\section{$B D A$ and $C B$ injection sites reconstruction}

The brain hemisphere injected with BDA (Table 1) was used to reconstruct the corresponding $\mathrm{BDA}$ injection site in $\mathrm{PM}$, SMA or 
TABLE 3. Quantitative data for the corticobulbar projection: number of axonal boutons

\begin{tabular}{|c|c|c|c|c|c|c|c|c|}
\hline & \multicolumn{3}{|l|}{ PM } & \multicolumn{3}{|l|}{ M1 } & \multirow{2}{*}{$\begin{array}{l}\text { SMA } \\
\text { SMA-1 }\end{array}$} & \multirow{2}{*}{$\begin{array}{l}\text { Spinal cord }(*) \\
\text { SC-1 }\end{array}$} \\
\hline & PM-1 & PM-2 & PM-3 & M1-1 & M1-2 & M1-3 & & \\
\hline Total number of boutons/neurons* & 6566 & 5070 & 2939 & 1013 & 217 & 1693 & 6035 & 2067 \\
\hline Ipsilateral boutons/neurons* & 3267 & 3004 & 1968 & 425 & 90 & 485 & 3225 & 1246 \\
\hline Contralateral boutons/neurons* & 3299 & 2066 & 971 & 588 & 127 & 1208 & 2810 & 821 \\
\hline \%global ipsilateral & 49.8 & 59.3 & 67.0 & 42.0 & 41.5 & 28.6 & 53.4 & 60.3 \\
\hline \%global contralateral & 50.2 & 40.7 & 33.0 & 58.0 & 58.5 & 71.4 & 46.6 & 39.7 \\
\hline Ipsilateral norm. number of boutons $(* 1000)$ & 1813 & 2039 & 1639 & 447 & 128 & 152 & 1493 & - \\
\hline Contralateral norm. number of boutons $(* 1000)$ & 1831 & 1403 & 809 & 619 & 181 & 378 & 1301 & - \\
\hline
\end{tabular}

The number of axonal boutons in PMRF (terminaux and en passant cumulated) is given in absolute number ( 3 top rows) and after normalisation based on the number of BDA labelled CST axons ( 2 bottom rows; see text). In SC-1 (rightmost column), the tracer CB was injected in the cervical cord (C5-C8) and the data are for the number of reticulospinal neurons in PMRF projecting to the cervical cord.

TABLE 4. Distribution in PMRF of the reticulospinal neurons as a result of $\mathrm{CB}$ injection in the cervical cord (C5-C8)

\begin{tabular}{|c|c|c|c|c|c|}
\hline & \multicolumn{5}{|l|}{ SC-1 } \\
\hline & $\begin{array}{l}\text { Tot. } \\
\text { Ipsilateral }\end{array}$ & $\begin{array}{l}\text { Tot. } \\
\text { Contralateral }\end{array}$ & $\begin{array}{l}\text { Total } \\
\text { both sides }\end{array}$ & $\begin{array}{l}\% \\
\text { ispilateral }\end{array}$ & $\begin{array}{l}\% \\
\text { contralateral }\end{array}$ \\
\hline $\mathrm{PnO}+\mathrm{PnC}$ & 196 & 205 & 401 & 48.9 & 51.1 \\
\hline $\mathrm{Gi}$ & 536 & 391 & 927 & 57.8 & 42.2 \\
\hline IRt & 190 & 50 & 240 & 79.2 & 20.8 \\
\hline LRt & 10 & 6 & 16 & 62.5 & 37.5 \\
\hline $\mathrm{VC}$ & 24 & 20 & 44 & 54.5 & 45.5 \\
\hline Others & 290 & 149 & 439 & 66.1 & 33.9 \\
\hline
\end{tabular}

M1 (Fig. 2A). The reconstruction was plotted on a lateral or top view of the injected hemisphere, taking into account anatomical references such as sulci. BDA was injected at multiple sites in each targeted motor cortical area. The BDA injection creates different areas known as 'core' and 'halo' (see Hamadjida et al., 2012); however, only in PM-1, PM-2, PM-3 and SMA-1 was it possible to make this differentiation. In M1-3, M1-2 and M1-1 no differentiation between 'core' and 'halo' was performed due to the difficulties to delimitate the two. The volume of the injection site was calculated using an ad-hoc function of the Neurolucida Explorer software based on the Cavalieri estimator (see Pizzimenti et al., 2007; Wyss et al., 2013 for a detailed description of the method).

The right cervical spinal cord was used to reconstruct the injection site of the retrograde tracer CB (Table 1, Fig. 2B). The reconstruction was drawn at a total magnification of $12.5 \times$ on the basis of tracer deposits. We could not differentiate between a 'halo' and a 'core' region.

\section{Results}

Anatomical studies of the corticobulbar projections using anterograde degeneration methods usually refer to the brainstem as medial and lateral tegmental fields (Kuypers, 1958, 1981). In the present study we considered the medial brainstem tegmentum as the medial part of the reticular formation comprising the Pontine reticular nucleus (Pars oralis and Pars caudalis; Pons), the Gigantocellular reticular nucleus (Medulla oblongata) as well as all nuclei located ventral and dorsal to the reticular formation. The lateral tegmental field here corresponds to the area lateral to the reticular nuclei cited above, comprising the Intermediate reticular nucleus and the Lateral reticular nucleus. Nuclei outside the reticular formation that are located ventral, dorsal or lateral to it belongs to the lateral part of the brainstem (Paxinos et al., 2000). At the caudal extent of the brainstem, we considered all the medial subdivisions of the reticular formation as Intermediate reticular nucleus (Paxinos et al., 2000). Figure 1 represents the extent of the PMRF nuclei and the location of the Raphe nucleus along the rostro-caudal extent of the brainstem.

Seven animals were injected with the anterograde tracer BDA in either PM, SMA or M1, whereas one additional monkey was injected with the retrograde tracer $\mathrm{CB}$ into the $\mathrm{C} 5-\mathrm{C} 8$ segments of the spinal cord. Figure 2 shows reconstructions of the BDA and CB injection sites for all animals. Three monkeys were injected in PM on the left hemisphere (PM-1, PM-2 and PM-3) whereas the remaining four were injected in M1 or SMA on the right hemisphere. The injection core was drawn in black and, when identifiable, the halo was drawn in grey. One monkey (SC-1) was injected with the tracer $\mathrm{CB}$ in the right $\mathrm{C} 5-\mathrm{C} 8$ segments of the spinal cord (same case reported in Rouiller et al. (1994) for the origin of the CS projection).

In PM, the BDA injections covered a large part of PMd and PMv in monkeys PM-1 and PM-3, whereas in monkey PM-2 BDA was restricted to PMd, also covering a large part of it (Fig. 2). In SMA and $\mathrm{M} 1$, in two monkeys the BDA injection was targeted to the hand area determined by ICMS (monkeys SMA-1 and M1-3 respectively). In monkey SMA-1, BDA was delivered along 3 vertical syringe penetrations, distant $1.5 \mathrm{~mm}$ apart along the rostro-caudal axis, at 2 sites along each penetration where $1.5 \mu \mathrm{l}$ was injected (4.5 and $5.5 \mathrm{~mm}$ deep with respect to the dura surface). In monkey M1-3, based on the ICMS map and the position of low threshold sites, BDA was injected mostly in the rostral bank of the central sulcus where the cortical layers are roughly perpendicular to the brain surface, thus corresponding to the 'new M1', as described by Rathelot \& Strick (2009). There were 3 syringe penetrations along the central sulcus ( $1 \mathrm{~mm}$ rostral to it), distant $1.1 \mathrm{~mm}$ from each other; BDA was injected at 2 or 3 depths along each penetration based on ICMS sites. The 'old M1' part, corresponding to the M1 territory where the cortical layers are parallel to the surface, was only partially included in the injection site (Fig. 2). In the other two monkeys injected in M1 (M1-1 and M1-3), the BDA injection was not restricted to the hand area (no ICMS) but also involved more proximal territories (located more medially). In both monkeys, 6 syringe penetrations perpendicular to the brain surface were performed along a fictive segment of $12.5 \mathrm{~mm}$ long in parallel to the central sulcus (1 $\mathrm{mm}$ more rostral), from the hand area laterally (at the medio- 
A
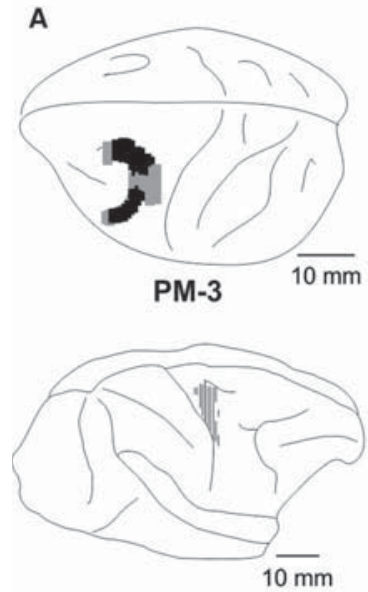

M1-1
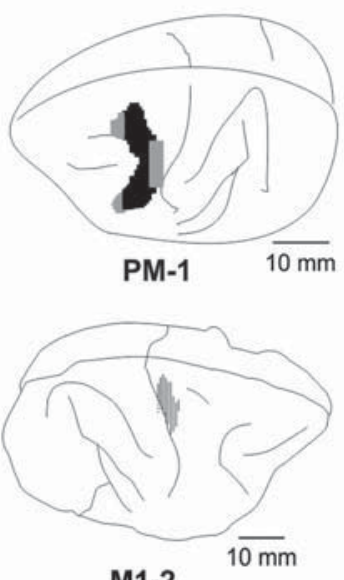

M1-2
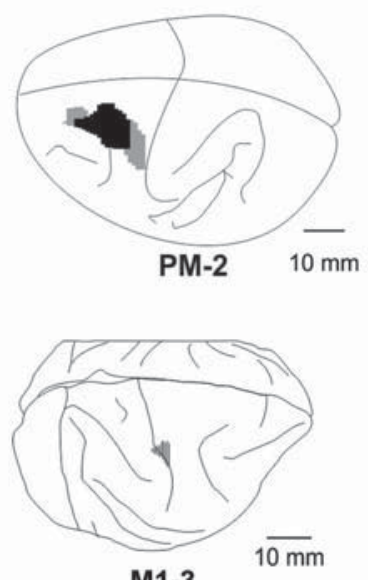

M1-3

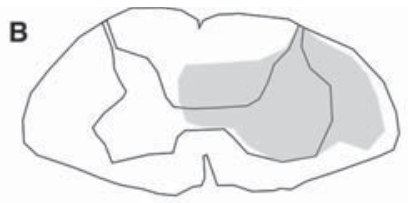

SC-1

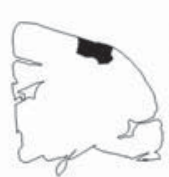

23b

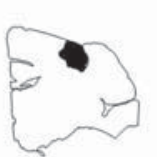

$28 b$

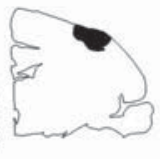

$24 b$

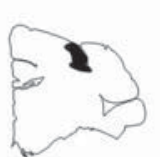

$29 b$

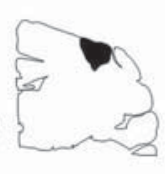

25b

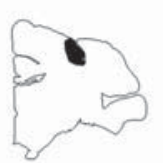

$30 \mathrm{~b}$

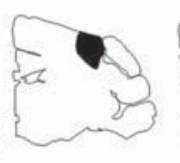

$26 b$

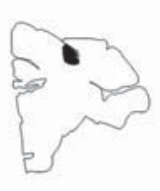

$31 b$

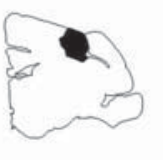

$27 b$

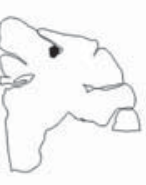

$32 b$

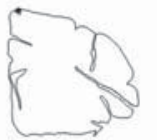

$12 b$

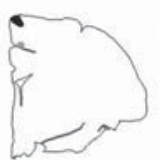

$17 b$

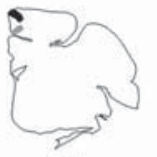

$13 b$

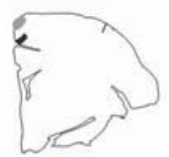

$18 b$

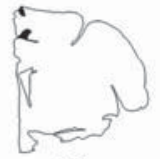

$14 \mathrm{~b}$

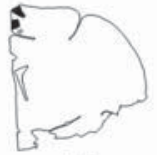

$15 b$

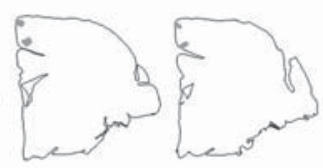

$20 b$

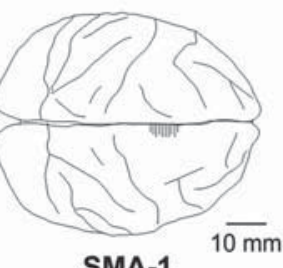

M1-3

$19 b$

SMA-1

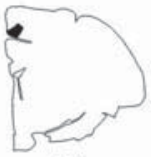

$16 \mathrm{~b}$

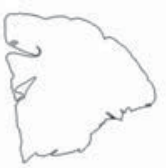

21b

FIG. 2. (A) Reconstruction of the BDA injection sites for each of the 7 monkeys. PM-3, PM-1, PM-2 were injected in the left hemisphere, whereas M1-1, M12, M1-3 and SMA-1 were injected in the right hemisphere. The injection core is represented in black whereas the halo is in grey (a halo was not present in all cases). Injections in SMA-1 and M1-3, at sites identified by ICMS, are in addition represented on alternate sections (1 out of 2). Sections are arranged from rostral to caudal following increasing sections numbers. (B) Reconstruction of the CB injection site on the right hemicervical spinal cord in SC-1.

lateral level of the genu of the arcutate sulcus) and then distant by $2.5 \mathrm{~mm}$ from each other going medially. Along each penetration, $1.5 \mu \mathrm{l} \mathrm{BDA}$ was delivered at 2 sites $(3$ and $7 \mathrm{~mm}$ deep from the dura surface) in order to fill the rostral bank of the central sulcus (new M1 area). In addition, 3 and 5 penetrations (in M1-1 and M12 respectively) were performed along a second segment parallel to the central sulcus but $3.5 \mathrm{~mm}$ more rostrally, with injection of BDA $(1.5 \mu \mathrm{l})$ at a single site $(3 \mathrm{~mm}$ deep) in order to include a part of the old M1.

Typical BDA or CB stained axon terminals or neurons, respectively, are illustrated in Fig. 3, with microphotographs representing BDA-labelled stem axons (Fig. 3A), BDA-labelled axonal terminal fields (Fig. 3B) and BDA labelled axonal boutons en passant or terminaux (Fig. 3C). Figure 3D represents CB-labelled reticulospinal neurons located in Gi nucleus. As far as axonal boutons are concerned, boutons en passant were well identified in most cases by the presence of a preceding and a continuing axon branch. In contrast, the distinction with boutons terminaux was not always clear, especially when boutons were located at the very top or very bottom of a section, without the possibility to check the presence of a preceding or a continuing axon branch on an adjacent section. For this reason, they were not charted with distinct markers (their proportion thus remains unknown). However, qualitatively, there was a clear predominance of boutons en passant as compared to terminaux.
The BDA labelling terminal axonal fields of the corticobulbar projection were mainly located in the nuclei of the PMRF, namely the Pontine reticular nucleus Pars oralis, the Pontine reticular nucleus Pars caudalis, the Gigantocellular reticular nucleus, the Intermediate reticular nucleus and the Lateral reticular nucleus (Fig. 4). As there were not always clear limits between some of the nuclei we grouped the two parts of the Pontine reticular nucleus $(\mathrm{Pn})$. Similarly, the Intermediate reticular nucleus and the Lateral reticular nucleus were fused (Rt). Outside the reticular formation, we analysed the corticobulbar projections in the Raphe nucleus (grouping the multiple Raphe subnuclei). The remaining boutons found in nuclei other than those listed above but identifiable were associated by default to the category 'other nuclei'. In contrast, the boutons located outside well defined nuclei were classified in the category 'undefined areas'. Both 'other nuclei' and 'undefined areas' categories were not included in the statistical analyses.

\section{Corticobulbar projections from the premotor cortex (PM)}

PM-1 and PM-3 were injected with BDA in both PMd and PMv whereas, in PM-2, BDA was injected in PMd only (Fig. 2). The typical bilateral distribution of BDA labelled stem axons and boutons in the brainstem is illustrated for 6 out of the 12 analysed 

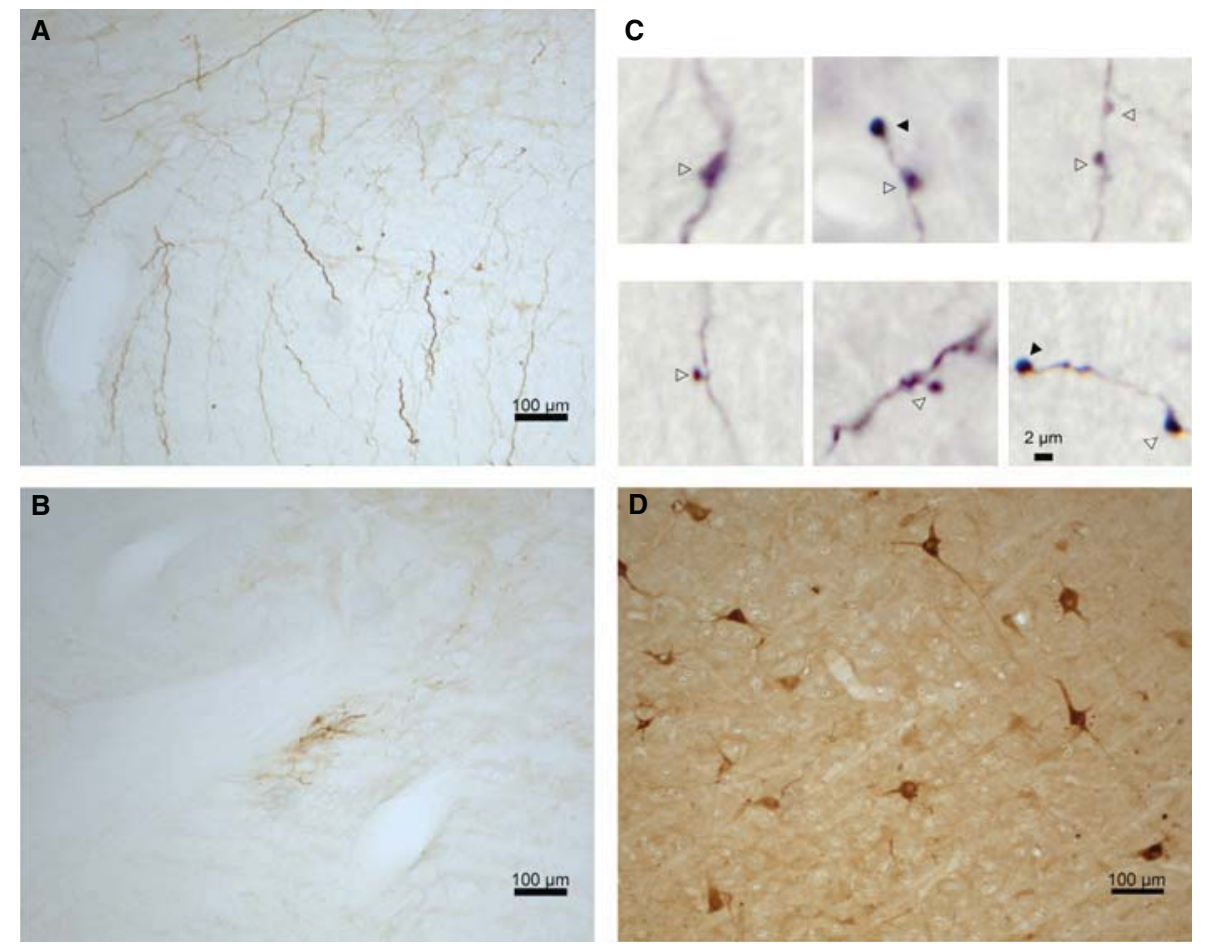

FIG. 3. (A-B) Microphotographs of PM-1 brainstem ipsilateral to the BDA injection site. (A) BDA-labelled stem axons; (B) BDA-labelled axonal terminal field; (C) Examples of BDA-labelled boutons en passant and terminaux in PMRF at high magnification, originating from SMA (case SMA-1). The open arrowheads point to boutons en passant, whereas the black arrowheads point to boutons terminaux; (D) Microphotograph representing reticulospinal neurons in the Gigantocellularis reticular nucleus labelled with CB injected in the cervical cord (case SC-1).

sections in PM-2 (Fig. 4A). On most sections BDA labelled boutons were found in the medial part of the PMRF, adjacent to the midline on both halves of the brainstem. The axonal boutons were found most abundantly in the nuclei $\mathrm{PnO}, \mathrm{PnC}$ and $\mathrm{Gi}$, bilaterally. PM-2 showed a statistically significant bilateral difference for its corticobulbar projection with a larger number of ipsilateral boutons in the rostral half of the brainstem when compared to the contralateral side $(P=0.048)$. However, there was no difference for the caudal half or when all sections were considered (Fig. 5A). Considering the distribution of boutons in the nuclei of the PMRF (Fig. 6), PM-2 exhibited a statistically significant bilateral difference in the Pontine reticular nuclei $(\mathrm{PnO}$ and $\mathrm{PnC})$ and in the Rt with more ipsilateral boutons in Pn $(P=0.004)$ and more contralateral boutons in Rt $(P=0.018)$. A significant difference was also found for the $\mathrm{Gi}$ nucleus $(P=0.008)$ with more boutons on the ipsilateral side. No statistically significant bilateral difference was found for the Raphe nucleus (Fig. 6).

PM-1 showed no differences in the number of BDA-labelled axonal boutons (both en passant and terminaux) between the ipsilateral and the contralateral sides of the brainstem (Fig. 5A). Considering the distribution of boutons across the nuclei of the PMRF (Pn, Gi and Rt) there was a statistically significant difference $(P=0.026)$ between the contralateral and ipsilateral sides for the pontine reticular nuclei $(\mathrm{PnO}$ and $\mathrm{PnC}$ ), with a denser corticobulbar projection on the ipsilateral side (Fig. 6). In contrast, there was no bilateral difference in the Gi, IRt and LRt nuclei. The same was true for the Raphe nucleus (Fig. 6).

In PM-3, there was a significant bilateral difference with a prevalence of ipsilateral boutons in the brainstem taken as a whole $(P=0.01)$. No difference was found in either the caudal or the rostral halves, although for the latter the three most rostral sections were not available (Fig. 5A), thus, no statistics have been performed between the ipsilateral and the contralateral number of boutons for the $\mathrm{PnO}$ and PnC nuclei. No significant difference was found for the Gi nucleus, whereas there was a significant bilateral difference with a prevalence of contralateral boutons $(P=0.008)$ for the Rt nuclei. No bilateral difference was found in the Raphe nucleus (Fig. 6).

Moreover, the distribution of the normalised numbers of boutons along the rostro-caudal axis in the two monkeys injected in PMd and PMv (PM-1 and PM-3) showed a peak of contralateral and ipsilateral distributions in the middle part of the PMRF (Fig. 7). A bilateral decrease in the number of boutons was observed at the most rostral and caudal parts of PMRF in PM-1 and at the caudal most part of PMRF in PM-3 on the contralateral side. For the latter, the three-first sections were not available. PM-2 (injection in PMd only) showed a denser distribution of boutons in the most rostral part of PMRF on the ipsilateral side. Along the rest of the PMRF the distribution profiles of contralateral and ipsilateral boutons were similar (Fig. 7).

The global percentages of ipsilateral vs. contralateral axonal boutons in the whole brainstem (Table 3) showed that, in PM-1, 50.2\% of the boutons were contralateral to the injection site, whereas $49.8 \%$ of the boutons were ipsilateral. Both PM-2 and PM-3 showed a global percentage of boutons higher on the ipsilateral side $(59.3 \%$ and $67 \%$ respectively) as compared to the contralateral side $(40.7 \%$ and $33 \%$ respectively).

\section{Corticobulbar projections from the primary motor cortex (M1)}

M1-1, M1-2 and M1-3 were injected with BDA in M1, although only M1-3 received a BDA injection restricted to the hand area (Fig. 2). M1-1 and M1-2 with larger BDA injections showed similar results, with a significantly larger number of corticobulbar boutons on the contralateral side, considering the whole brainstem 
A
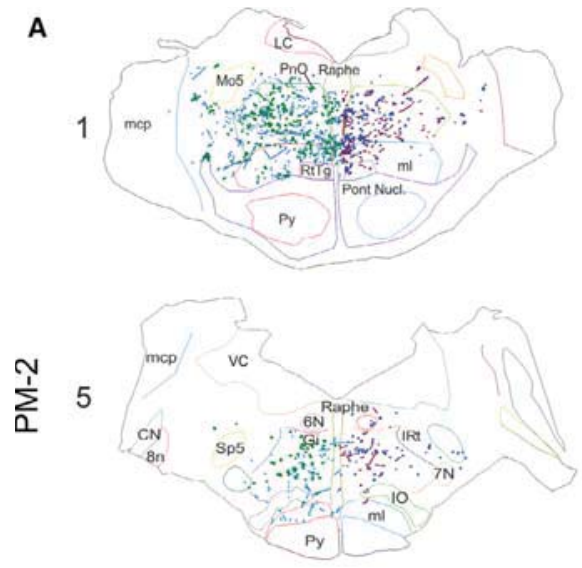

9

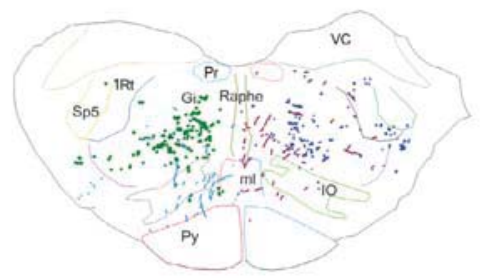

B
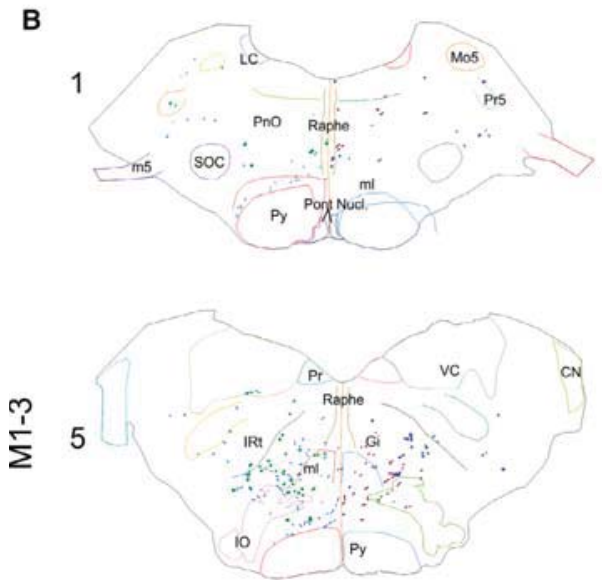

9

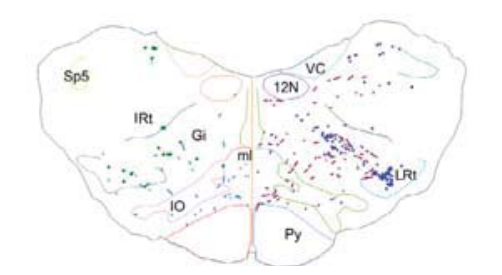

3

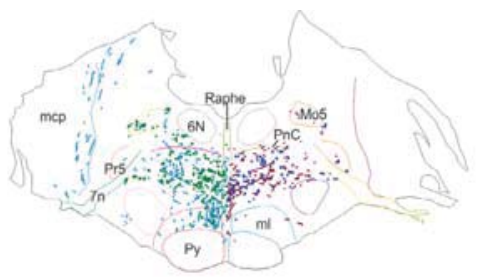

7

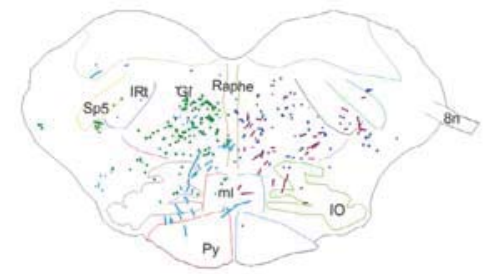

11

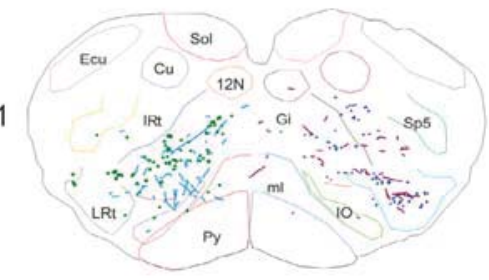

CN

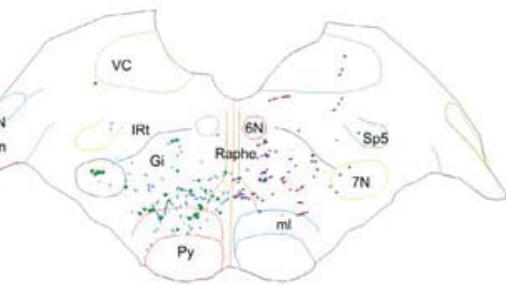

7

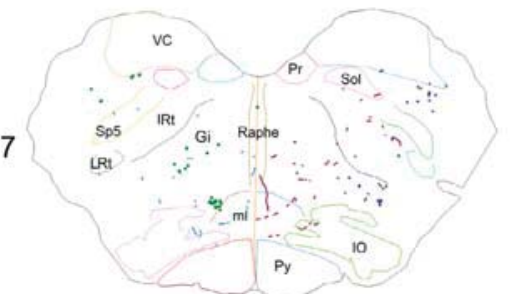

11

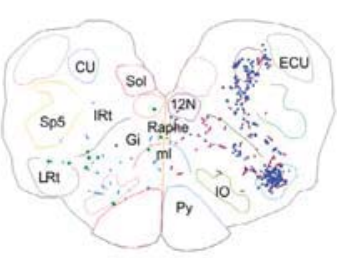

FIG. 4. Reconstructions of brainstem coronal sections of PM-2 (A) and M1-3 (B), arranged from rostral (section 1) to caudal (section 11). As in the other monkeys, overall 12 sections were analysed along the rostrocaudal axis of PMRF (see Fig. 1); however, one section every two of the 12 analysed sections are shown here (namely the odd numbers 1, 3, 5, 7, 9 and 11). The nuclei were identified based on the Paxinos et al. atlas (2000). BDA-labelled stem axons located ipsilateral to the BDA injection are marked in blue whereas those located contralateral are marked in bordeaux. BDA-labelled boutons en passant and terminaux, ipsilateral to the BDA injection are marked as green circles whereas the contralateral boutons are marked as blue squares. BDA was injected in PM in PM-2 and in M1 in M1-3.

( $P=0.003$ and $P=0.040$ respectively) or the rostral half of the brainstem $(P=0.015$ and $P=0.017$ respectively; Fig. 5A). In both animals no bilateral difference was found in the caudal half (Fig. 5A) and when considering the various nuclei of the PMRF or the Raphe nucleus (Fig. 6).

M1-3 showed a significant bilateral difference in the caudal half of the brainstem $(P=0.040)$ with more contralateral axonal boutons. No difference was found for the whole brainstem as well as for the rostral half (Fig. 5A). Considering the distribution of boutons in the PMRF nuclei, the Pn nuclei and the Gi nucleus did not show any bilateral difference, in contrast to the nuclei Rt $(P=0.039)$ with more boutons on the contralateral side. The raphe nucleus showed no bilateral difference (Fig. 6). In all three cases subjected to BDA injection in $\mathrm{M} 1$, it appeared that the corticobulbar terminals tended to be more numerous laterally than medially, especially in the most caudal sections (Fig. 4B). 

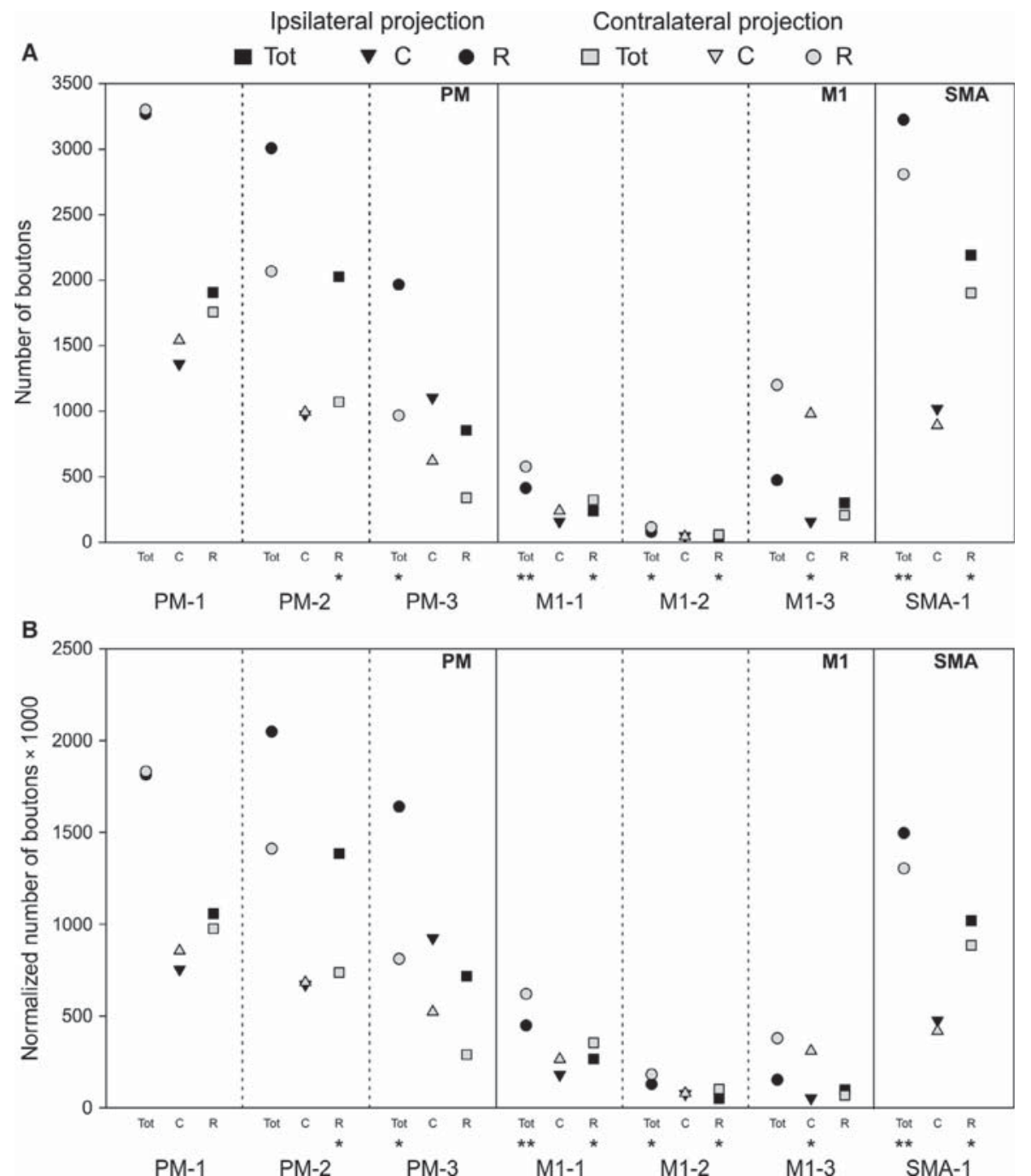

FIG. 5. Scatter plots showing for each monkey the total number of boutons en passant and terminaux in the whole brainstem (Tot), in its caudal half (C; from section 7 to section 12) and in its rostral half (R; from section 1 to section 6). Black symbols are for ipsilateral projections and grey symbols for contralateral ones. Data represent the numbers of boutons found in the entire brainstem, namely the nuclei of the PMRF, Raphe, all the other nuclei as well as all those found in undefined areas. (A) Scatter plots represent the raw data (absolute numbers of boutons), (B) Scatter plots showing the same data as in A but normalised according to the number of BDA labelled corticospinal axons observed above the Decussatio pyramidum. The cortical area of BDA injection is shown on the right corner of each panel. Statistically significant comparisons (ipsilateral vs. contralateral) derived from the Paired $t$-test/Wilcoxon test are represented with asterisks: ${ }^{*} P \leq 0.05 ; * * P \leq 0.01$.

The distributions of the normalised number of boutons along the rostro-caudal axis for M1-1 and M1-2 (injection in M1) were similar on both the ipsilateral and the contralateral sides along the rostro-caudal axis of the brainstem. Only M1-3 showed a higher number of boutons on the contralateral side of the caudal part of the PMRF as compared to the ipsilateral side (Fig. 7). All three animals injected in M1 showed a higher global percentage of boutons on the contralateral side of the brainstem (Table 3), ranging ipsilaterally from $29 \%$ to $42 \%$ and contralaterally from $58 \%$ to $71 \%$.

\section{Corticobulbar projections from the supplementary motor cortical area (SMA)}

SMA-1 was injected with BDA in the hand area of SMA (Fig. 2). SMA-1 showed a significant bilateral difference in both the whole brainstem $(P=0.008)$ and its rostral half $(P=0.040)$, biased towards the ipsilateral side. No bilateral difference was found in the caudal half (Fig. 5A). SMA-1 showed no bilateral difference between the ipsilateral and the contralateral sides for the Pn nuclei, Rt nuclei as well as the Raphe nucleus. In contrast, a significant bilateral difference was found for the Gi nucleus, biased towards the ipsilateral side $(P=0.027)$ (Fig. 6). For SMA-1 the distribution of normalised number of boutons along the rostro-caudal axis showed a similar bilateral distribution with more boutons in the rostral part of the PMRF than caudally (Fig. 7). The global percentages of ipsilateral vs. contralateral boutons in the whole brainstem (Table 3) for SMA-1 showed a percentage of $53.4 \%$ ipsilaterally and $46.6 \%$ contralaterally. As for PM, the corticobulbar projection originating from SMA terminated predominantly in the medial part of PMRF (not shown).

\section{Normalised data}

Due to variations across animals with respect to the total volume of BDA injected, as well as the number of infusion sites 
PM
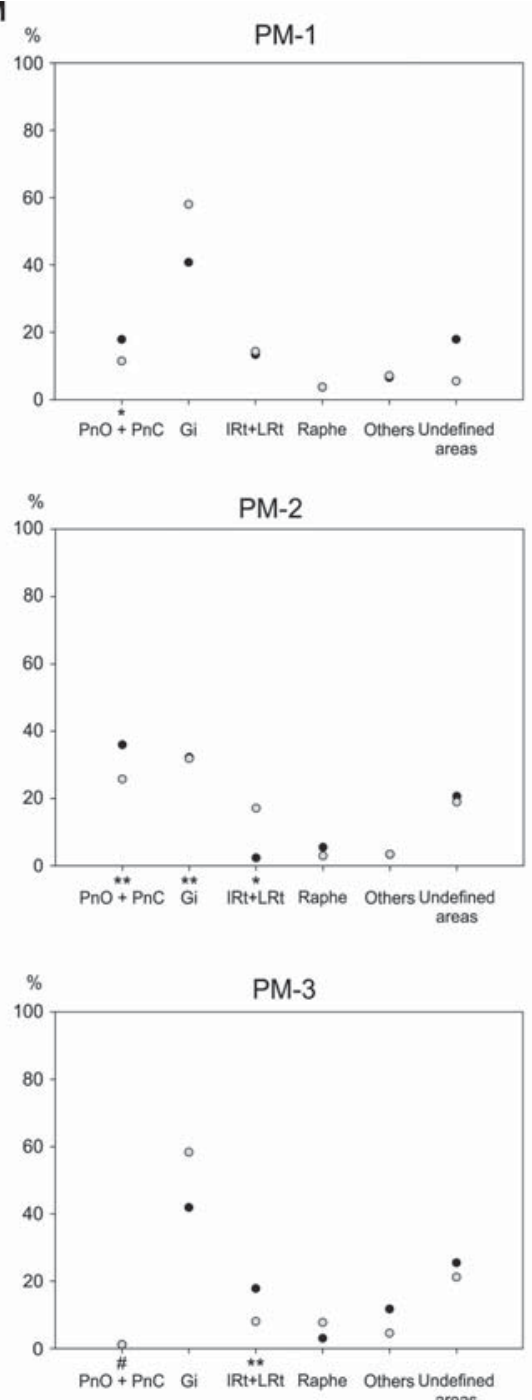

M1
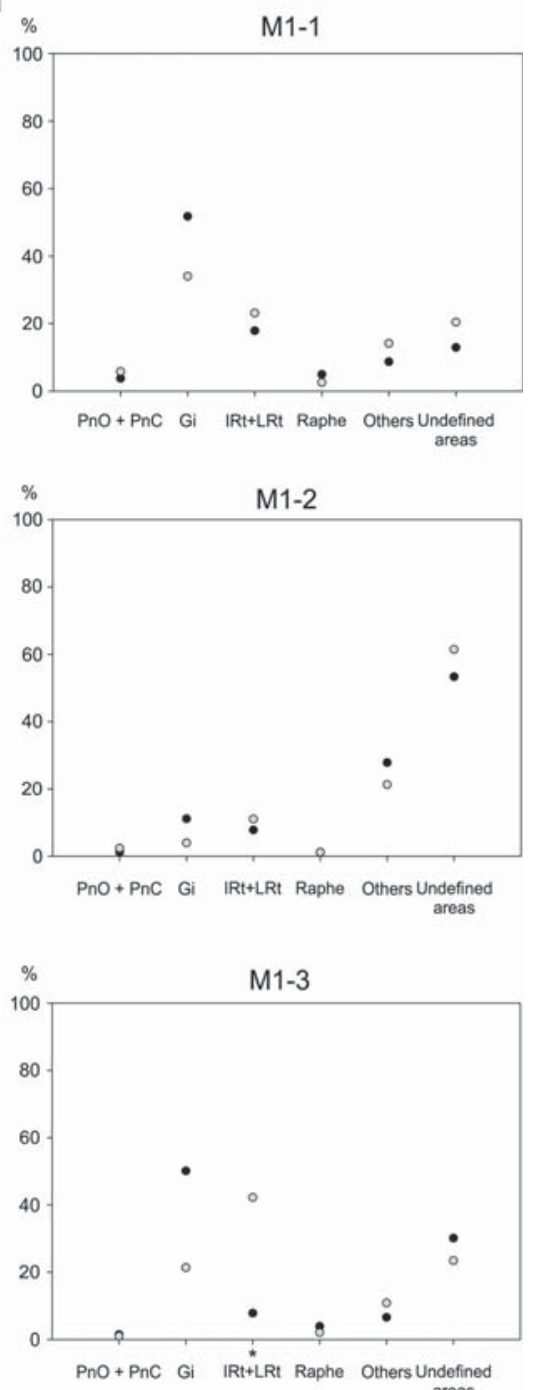

SMA

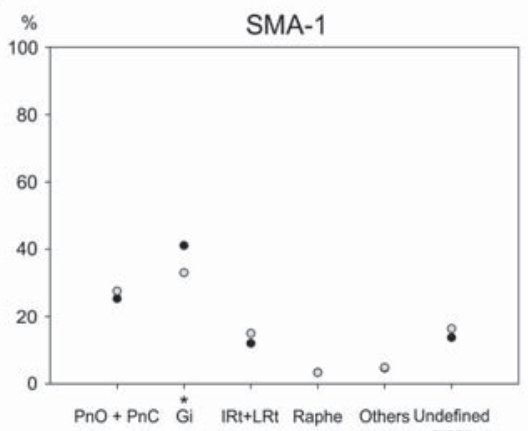

Ipsilateral projection

Contralateral projection

FIG. 6. Distribution of BDA-labelled axonal boutons across brainstem nuclei in each monkey. Percentages of boutons en passant and terminaux ipsilateral (black) and contralateral (grey) calculated on the total numbers of boutons found in the whole brainstem ipsilateral or contralateral to the injection site. The left panels show data obtained from animals injected in PM. In PM-1 and PM-3 the BDA injection was located in both PMd and PMv, whereas in PM-2 the injection was restricted to PMd. The right panels show data obtained from animals injected in M1. In M1-1 and M1-2 the injection was larger than that performed in M1-3, which was restricted to the hand area. The bottom panel shows data obtained from the single animal injected in SMA, in the hand area. Statistically significant bilateral comparisons (ipsilateral vs. contralateral and for each nucleus (nuclei)) derived from the Paired $t$-test/Wilcoxon test are represented with asterisks along the abscissa: ${ }^{*} P \leq 0.05 ; * * P \leq 0.01, * * * P \leq 0.001$. The black/grey symbols without "** do not show a statistically significant bilateral difference. In each graph, the sum of all black symbols is $100 \%$. The same for the grey symbols (100\%). The "\#” indicates the absence of the corresponding sections. 
PM

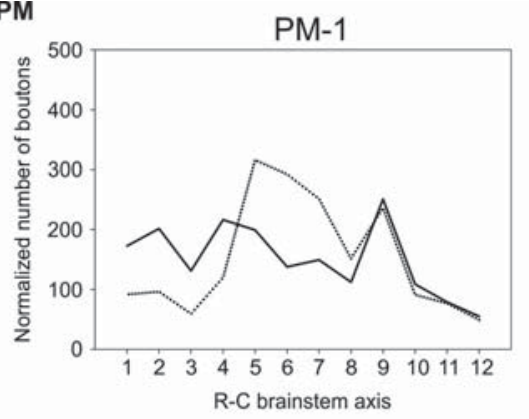

PM-2

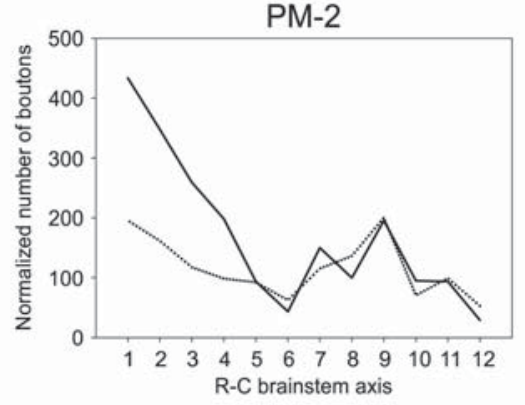

PM-3

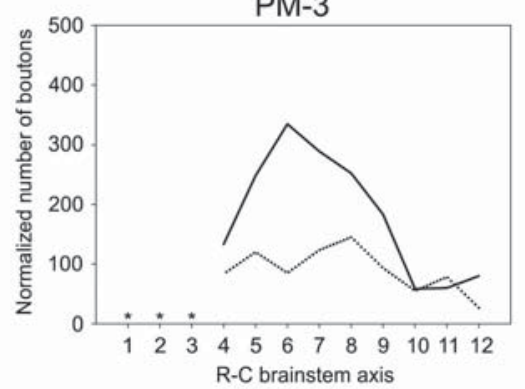

SMA

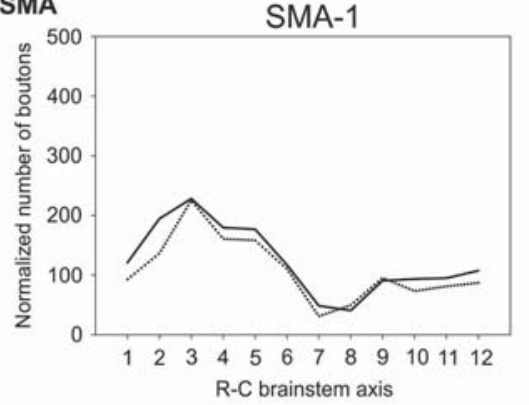

M1

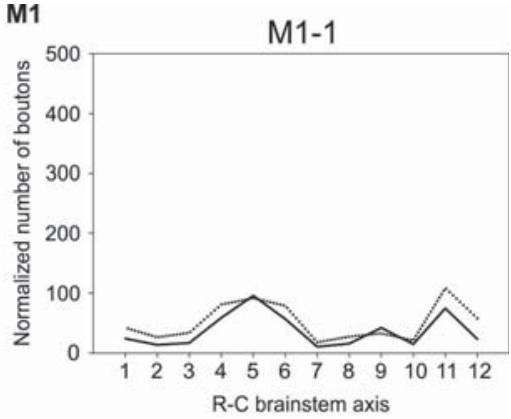

M1-2

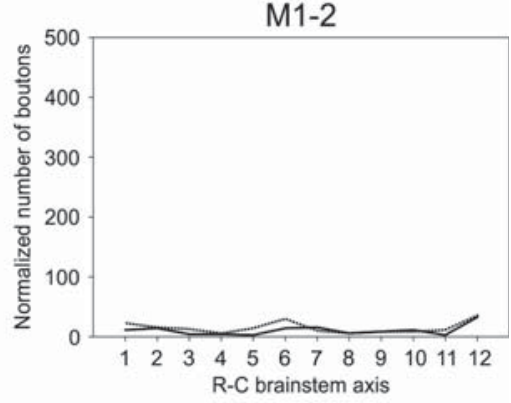

M1-3

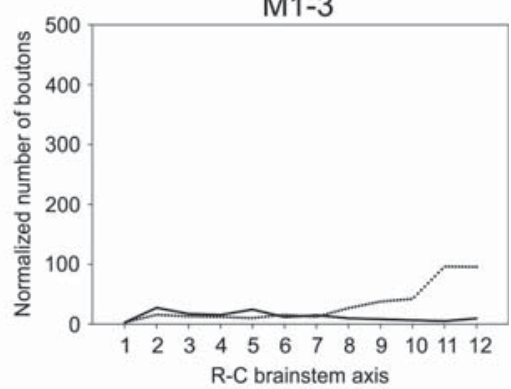

C5-C8
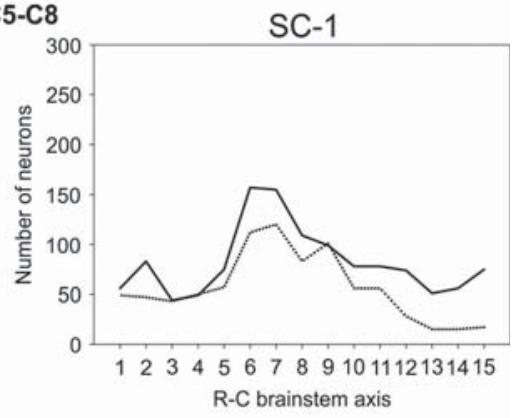

Contralateral projection

FIG. 7. Distribution of BDA-labelled axonal boutons (PM, SMA, M1) or reticulospinal neurons (SC-1) along the rostro-caudal (R-C) brainstem axis. The number of boutons was normalised with respect to the number of CS axons above the pyramidal decussation. See also legend of Fig. 6 . The '*' indicates the absence of the corresponding sections. The ordinate scale is different in the bottom right panel as its concerns reticulospinal neurons whereas all other panels show the distribution of corticobulbar boutons.

(Table 1), the BDA injection sites exhibit variable volumes (Fig. 2). As a result, a direct comparison of the number of corticobulbar boutons across animals is likely to be biased (Fig. 5A). To allow a more meaningful inter-individual comparison, the number of BDA-labelled corticospinal (CS) axons was established on a section located just above the pyramidal decussation (Table 1). The number of CS axons was then used to normalise the data: the number of corticobulbar boutons (Fig. 5B) was divided by the number of CS axons *1000. Using CS axons as a reference for normalisation is consistent with the observation that at least a part of the corticobulbar terminals arise from collaterals of the CST (Keizer \& Kuypers, 1989). As a consequence, one may expect that the more CS axons labelled with BDA, the more projections will terminate in the brainstem.

After normalisation based on the number of CS axons (Table 1), it appeared that more corticobulbar axonal boutons arise from PM and SMA than from M1 (Fig. 5B; Table 3). Moreover, the corticobulbar projection originating from PM and SMA was stronger ipsilaterally whereas that from M1 terminate more predominantly on the contralateral side. 
SC-1
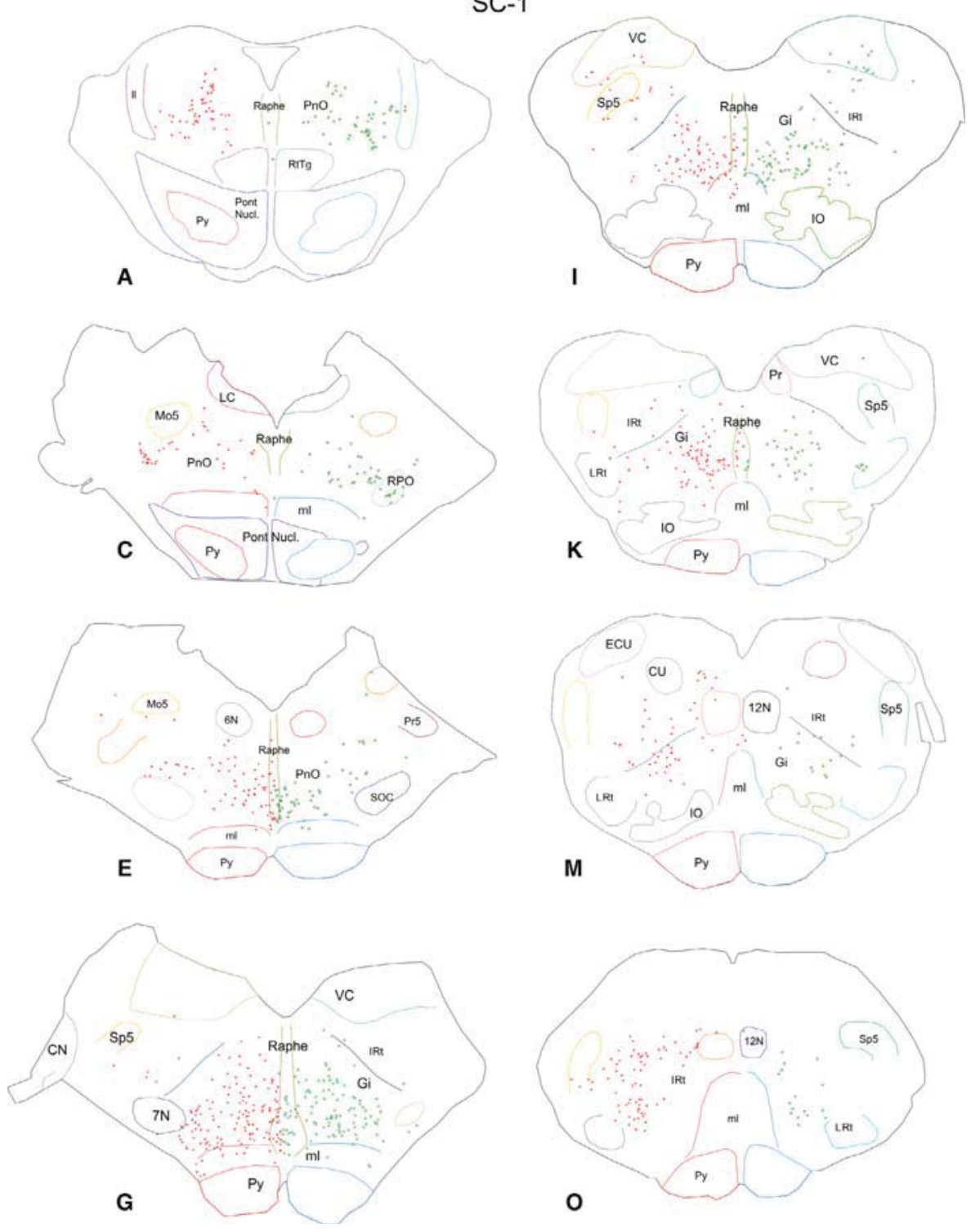

FIG. 8. Reconstruction of brainstem sections ( 15 sections analysed from rostral to caudal, 'A' to 'O') representing the distribution of reticulospinal neurons on the ipsilateral (red) and the contralateral sides (green) as a result of injection of the retrograde tracer CB in C5-C8 hemi-spinal cord (case SC-1). In the Figure, only one every two sections is represented (A, C, E, G, I, K, M, O).

\section{Origin of the reticulospinal projection to the cervical cord (C5- C8)}

SC-1 was injected unilaterally into segments $\mathrm{C} 5-\mathrm{C} 8$ of the spinal cord with the retrograde tracer $\mathrm{CB}$. The tracer marked reticulospinal neurons into the PMRF (Figs 3D and 8). We found that the area of distribution of reticulospinal neurons in PMRF largely matched to the nuclei of PMRF containing the axonal boutons of the corticobulbar projections (Figs 4 and 8). Figure 7 (bottom right panel) shows the rostrocaudal distribution of the reticulospinal neurons in PMRF, with predominance on the ipsilateral side with respect to the $\mathrm{CB}$ injection, although reticulospinal neurons were found bilaterally. The global percentages of $\mathrm{CB}$ labelled reticulospinal neurons (Table 3) for SC-1 showed a percentage of $60.3 \%$ ipsilaterally and $39.7 \%$ contralaterally with respect to the injected segments of the spinal cord.

Table 4 shows the percentage of reticulospinal neurons in each PMRF nucleus on both ipsilateral and contralateral sides with respect to the injection of $\mathrm{CB}$ tracer. In $\mathrm{Pn}$ nuclei $(\mathrm{PnO}$ and $\mathrm{PnC}$ together), $48.9 \%$ of neurons were ipsilateral whereas $51.1 \%$ were contralateral to the injection site in the spinal cord. We did not separate the two nuclei as $\mathrm{PnC}$ was present in one section only. The nucleus Gi exhibited a percentage of $57.8 \%$ of ipsilateral and $42.2 \%$ of contralateral reticulospinal neurons. For IRt and LRt reticulospinal neurons showed a percentage of $79.2 \%$ and $62.5 \%$ ipsilaterally, respectively, and $20.8 \%$ and $37.5 \%$ contralaterally respectively.

\section{Discussion}

In summary, the present data largely confirm our hypothesis that the corticobulbar projection on PMRF in macaques differ depending on the motor cortical area of origin (PM, SMA or M1). First of all, based on both raw and normalised data, the corticobulbar projection is denser when originating from PM or SMA as compared to that 
arising from M1 (Fig. 5; Table 3): quantitatively, there were at least twice as many corticobulbar axonal boutons when originating from PM or SMA than from M1. Second, corticobulbar axonal boutons originating from M1 were more numerous on the contralateral side (58-71\%) than ipsilaterally (29-42\%), whereas the corticobulbar projections from PM and SMA were generally ipsilateral predominant (Fig. 6; Table 3). Third, although the nucleus Gi is a major target of the corticobulbar projection, irrespective of the motor cortical area of origin, the corticobulbar projections originating from PM and SMA gave rise to more axonal boutons in the nuclei PnO and $\mathrm{PnC}$ than when originating from M1 (Fig. 6). Fourth, the corticobulbar projections from PM and SMA terminate predominantly in the medial part of PMRF (mPMRF), whereas the projections from M1 terminate more laterally in PMRF, mainly in the caudal half of the brainstem; more rostrally, the projection from M1 is less dense and is located in mPMRF.

The raw and normalised quantitative data yielded consistent results (Fig. 5), reinforcing the conclusion that the corticobulbar projection is denser when originating from non-primary motor cortical areas than from M1. On the one hand, the normalisation procedure based on the number of CS axons, which originate from cortical layer $\mathrm{V}$ as do the corticobulbar axons, may prevent to some extent an inter-individual bias due to variability on how much of layer $\mathrm{V}$ is included in the BDA injection site. Such variability is reflected by the large range in the number of BDA labelled CS axons across monkeys (Table 1). The raw data of Fig. 5A showing a low density corticobulbar projection from M1 may be due to BDA spread invading layer $\mathrm{V}$ to a lesser extent in these animals, which may be the case in monkeys M1-1 and M1-2 (Table 1). In such a case, the normalisation procedure is likely to correct this bias, at least to some extent. On the other hand, such normalisation has clear limitations. In particular, the number of CS axons used for normalisation varies across motor cortical areas of origin (Dum \& Strick, 1991, 1996; He et al., 1993, 1995). As a consequence, similar BDA injection sites may label a proportionally larger portion of SMA and PM than M1, leading to an overrepresentation of CS axons from PM and SMA and an underrepresentation from M1. Moreover, only a fraction of corticobulbar projections to PMRF are indeed collaterals of CS axons (Keizer \& Kuypers, 1989) and therefore the normalisation procedure based on CS axons may not be as adequate for non-collateral corticobulbar projections. In other words, normalisation may also introduce bias and should therefore be interpreted cautiously. In the present case, at least the normalised data do not challenge the raw data, as both types of data consistently support the density difference across motor cortical areas with respect to their corticobulbar projection.

A comparison with the literature on the corticobulbar projection in non-human primates needs to distinguish early studies based on the mostly semi-qualitative methods of anterograde degeneration (Kuypers, 1958; Kuypers \& Lawrence, 1967; see also review by Lemon, 2016) from more recent studies using modern neuroanatomical tracers, allowing better visualisation of axonal terminal fields (including individual boutons) and more precise quantification. Early studies, involving a large number of animals, have shown that the corticobulbar projections originating from PM (both dorsal and ventral) as well as from SMA terminate in the medial reticular formation (Keizer \& Kuypers, 1989; Kuypers \& Lawrence, 1967; see also Lemon, 2016), a location confirmed in the present study (Fig. 4A). Furthermore, Kuypers \& Lawrence (1967) have reported that the projections arising from the precentral gyrus (corresponding to our M1 injections) preferentially terminate in the lateral part of the brainstem, an observation also confirmed here (Fig. 4B), although the projection from M1 also spreads medially in Gi, especially in the rostral half of the brainstem. More recent studies derived from 'modern' neuroanatomical tracers, though restricted to a smaller number of animals, allowed for a refinement of the properties of the corticobulbar projection in monkeys. Keizer \& Kuypers (1989), using a double retrograde fluorescent labelling technique, showed that the CST projection gives rise to axon collaterals terminating in the medial part of the brainstem. One tracer was injected unilaterally in the cervical cord (C2) and a second tracer ipsilaterally in the bulbar reticular formation. The authors estimated that the proportion of double labelled cells in the hemisphere opposite to the injections ranged from $10 \%$ to $30 \%$ of the total number of labelled neurons (single and double labelling). These double labelled cells correspond to branching neurons projecting to both the bulbar reticular formation and the cervical cord contralaterally. Such double labelled neurons were reported to be present in the motor cortical areas investigated here, namely M1, SMA and PM. The proportion of branching neurons is not known for the ipsilateral corticobulbar projection. Using BDA as anterograde tracer, Borra et al. (2010) have demonstrated that the PMv hand area, besides its CS projection, gives rise to a corticobulbar projection terminating in the main nuclei of the PMRF, namely Pn and Gi nuclei, in line with our PM cases involving PMv (PM-1 and PM-3; see our Fig. 6). As far as $\mathrm{PM}$ is concerned, the present data extend the same observations to PMd (our case PM-2 with an injection aimed at PMd: see our Fig. 6).

An original observation of this study, resulting from a quantitative comparison of the three motor cortical areas and based on both raw and normalised data, is that the corticobulbar projection is denser when originating from PM and SMA than from M1. Nevertheless, the fairly moderate corticobulbar projection from M1 may apply essentially for the caudal part of M1 ('new M1' as defined by Rathelot \& Strick, 2009), where BDA was mostly injected in our 3 M1 cases (Fig. 2). The situation may be different for the corticobulbar projection originating from the rostral part of M1 ('old M1' of Rathelot \& Strick, 2009), less affected by our BDA injections. All individual corticobulbar boutons could be charted in the present study, as this projection appears moderately dense, far below the density of the corticofugal projections directed towards the red nucleus and the pontine nuclei for instance. For this reason, it was not appropriate to use a stereological approach, also because it was not our goal to determine the absolute total number of corticobulbar boutons, but limit our analysis to a relative comparison between the three motor cortical areas of origin. Functionally, the denser corticobulbar projection from non-primary motor cortical areas, as compared to weaker projections from M1, suggest that the reticulospinal projection system is in position to receive prominent commands from PM and SMA related to preparation and planning of the voluntary movements, before delayed and less influential execution commands originating from M1 arrive to PMRF. In such a way, the reticulospinal system may better adjust posture for instance before execution of the intended voluntary movements. From the dense corticobulbar projection observed from PM and SMA, one may speculate that it may also be the case from the projection originating from another non-primary motor area, the cingulate motor cortex. A hypothesis to be tested in the future, based on specific injection in that cortical motor area.

Our data obtained from retrograde tracer injections in the cervical spinal cord segments $\mathrm{C} 5-\mathrm{C} 8$ of SC-1 confirmed that reticulospinal neurons are located in the nuclei of the PMRF where we found corticobulbar terminals (Fig. 3 and 4). Sakai et al. (2009) observed in macaque monkeys a split of $60-40 \%$ of reticulospinal neurons in 
the ipsilateral-contralateral side in Gi nucleus, respectively. Our data are in agreement with theirs, as we found a bilateral distribution of reticulospinal neurons in the Gi nucleus of $58-42 \%$ ipsilateral-contralateral (Table 4). However, some differences were present when we consider the Pn nuclei. We observed that in $\mathrm{PnO}$ and $\mathrm{PnC}$ together, there were nearly as many ipsilateral and contralateral reticulospinal neurons in PMRF (49-51\%; Table 4). With regards to Pn nuclei, Sakai et al. (2009) found that $\mathrm{PnO}$ had a contralateral projection bias of $40-60 \%$, whereas PnC projected mainly bilaterally (50-50\%). These bilateral differences relating to reticulospinal neurons in Pn nuclei may be explained by variations in segmental injection. We injected CB into more caudal cervical segments (C5-C8) of the spinal cord than their injections located in more rostral (C4-C5) spinal cord segments. Moreover, we analysed a greater number of sections along the rostro-caudal axis (15 sections) at a magnification of $100 \times$ on a frontal plane compared to the 4 parasagittal sections analysed in the study of Sakai et al. (2009) at magnification of 200-400×. The lateral reticular formation (Rt nuclei) showed mainly an ipsilateral predominance of retrogradely stained reticulospinal neurons (79-21\% in IRt nucleus and $63-37 \%$ in LRt nucleus) (Table 4). The present data allow to compare in the same species of macaque the corticobulbar projection with its terminal zone in the brainstem with the origin of the reticulospinal tract, with emphasis on the hand representation (Figs 4 and 8; Fig. 7). Indeed, the reticulospinal neurons shown in Fig. 8 project to segment $\mathrm{C} 5-\mathrm{C} 8$, where most hand muscle motoneurons are located (Jenny \& Inukai, 1983). The corticobulbar projection whose termination zone is described here originates from the hand area in M1-3 (M1) and in SMA-1 (SMA) or from larger cortical areas (five other monkeys) but including hand representation in M1 (M1-2, M1-1) or in PMv (PM-3, PM-1). The match of corticobulbar terminal and reticulospinal neurons in the nuclei Pn and Gi mostly (Figs 4 and 8) suggest that they represent sites where the motor cortical areas M1, PM and SMA may exert an influence on the RST in relation of the control of distal forelimb muscles, in line with previous evidence (Honeycutt et al., 2013; Riddle \& Baker, 2010; Riddle et al., 2009; Soteropoulos et al., 2012).

LRt neurons (LRN) are a convergent centre for motor information coming from the interneuronal systems of the spinal cord and from supraspinal centres as the motor cortex, the red nucleus, the superior colliculus and the trigeminal system (Alstermark et al., 1981; Alstermark \& Ekertot, 2013; Alstermark \& Ekerot, 2015). LRN then sends projections directly to the cerebellar cortex and via collaterals to the deep cerebellar nuclei (Alstermark \& Ekerot, 2015). Thus, the information coming from the spinal cord interneurons to the cerebellum via LRt may be used to modify and adjust the cerebellar output for reaching, grasping and postural movements (Alstermark et al., 1981; Alstermark \& Ekerot, 2015). Moreover, it has been demonstrated in cats and monkeys that corticoreticular projections on LRt follow a somatotopic organization (Marini \& Wiesendanger, 1987; Wiesendanger \& Wiesendanger, 1987; Rho et al., 1997). Previous studies on monkeys have shown that cortical projections to LRt nucleus arise mainly from the contralateral M1 and only partly from PM (Marini \& Wiesendanger, 1987; Wiesendanger \& Wiesendanger, 1987). Our results are in agreement with these previous studies. In general projections from the motor cortex on this nucleus were bilateral but with a tendency to end mainly on the contralateral side of the PMRF. However, only M1-3 showed a statistically significant difference for the contralateral side after BDA injection in the hand area of M1, indicating stronger projections onto this nucleus. These results are in agreement with previous studies (Kuypers, 1958; Marini \& Wiesendanger, 1987; Wiesendanger \& Wiesendanger, 1987; Matsuyama \& Drew, 1997) showing that forelimb regions of M1 are strongly connected to the contralateral LRt nucleus. These projections may inform the LRt nucleus about the ongoing descending motor commands so that it can integrate it with the information coming from other parts of the nervous system and then place the cerebellum in position to generate quick adjustments (Alstermark \& Ekertot, 2013; Alstermark \& Ekerot, 2015). The neurons of LRt could thus be seen as an integration centre for ascending and descending motor information directed to both the cerebellum and brainstem pathways.

We also found corticobulbar projections on the Raphe nucleus, which is composed by several nuclei located in distinct regions along the entire rostro-caudal extent of the brainstem and whose cytoarchitectonic limits are scarcely defined (Paxinos et al., 2000; Hornung, 2003). They contain heterogeneous populations of neurons with a majority of serotonergic (5-HT) neurons, making the Raphe nuclei the main centre of serotonin production for the entire brain (Hornung, 2003; Myers et al., 2016). The nuclei composing the entire Raphe nucleus can be subdivided into an ascending group and a descending group (Hornung, 2003; Myers et al., 2016). The latter is at the origin of the raphespinal tract projecting to the spinal cord (Perrier \& Cotel, 2015; Hornung, 2003; Myers et al., 2016; : Kuypers, 1981), divided into two pathways: a ventromedial pathway projecting to the intermediate and ventral horns (for autonomous and motor functions, respectively) and a dorsolateral pathway projecting to the dorsal horn (role in pain modulation) (Jacobs \& Azmitia, 1992; Hornung, 2003). Serotonin appears to have a role in motor modulation at the spinal cord level (Perrier \& Cotel, 2015). Using retrograde techniques, it was discovered that Raphe nuclei receive projections from the forebrain and hypothalamic areas, mainly involved in the limbic system. Scarce projections on Raphe nuclei from other brainstem nuclei were also found (Behzadi et al., 1990). We found, although fairly weak (Fig. 6), bilateral projections from M1, PM and SMA onto Raphe nuclei. To the best of our knowledge, no other studies have reported these connections yet, which are in position to influence the Raphe nuclei and the corresponding serotonergic system vis a vis motor planning and action, in order to appropriately regulate the delivery of serotonin at spinal cord level.

Functionally, in the control of voluntary movements the M1 hand area is a strongly lateralised system, exerting its effects mostly on the opposite distal forelimb. In contrast, SMA and PM appear to be more bilaterally organised, especially SMA (e.g. Wiesendanger et al., 1996; Kermadi et al., 1998, 2000; Kazennikov et al., 1999). A somewhat similar difference with respect to bilaterality appeared here for the corticobulbar projection. When originating from M1, the corticobulbar projection was predominantly crossed, with a majority of axonal boutons in the contralateral brainstem (Table 3: $58-71 \%)$. The corticobulbar projection from SMA was roughly equal on both sides (in a single animal), with a slight ipsilateral dominance (53\%). When originating from PM, the density of the corticobulbar projection was similar on both sides in one monkey and predominant ipsilaterally in two monkeys (59 and 67\%).

Voluntary independent finger movements are mostly under the control of the corticospinal tract through its direct connection on hand motoneurons referred to as the corticomotoneuronal (CM) system, a prerogative of primates (Lawrence \& Kuypers, 1968a,b; Lemon, 1993, 2008, 2010; Lemon \& Griffiths, 2005; Courtine et al., 2007; Schieber, 2007; Isa et al., 2013). These corticospinal projections arise from several areas of the frontal lobe (M1, SMA, PM and cingulate motor areas) as well as from the parietal lobe (Murray \& Coulter, 1981; Dum \& Strick, 1991, 1996, 2002; He et al., 1993, 1995; Chouinard \& Paus, 2006). In the frontal lobe, the number of CST projections arising from premotor cortical areas is roughly 
equal to those arising from M1 (Dum \& Strick, 1991, 2002; He et al., 1993). Following lesion of the CST/CM systems a flaccid paralysis of the hand occurs together with a loss of manual dexterity (Liu \& Rouiller, 1999; Courtine et al., 2007; Lemon, 2008; Darling et al., 2009, 2014; Kaeser et al., 2010, 2011; Bashir et al., 2012; Hamadjida et al., 2012; Zaaimi et al., 2012; Hoogewoud et al., 2013; Baker et al., 2015). With the aim to restore functional recovery after lesion of either the CST/CM or of the cerebral cortex (e.g. stroke) the reticulospinal tract (RST) may be considered as a possible anatomical substitute underlying incomplete recovery due to its role in the control on muscles involved in reaching and hand movements (Davidson \& Buford, 2004; Zaaimi et al., 2012; OrtizRosario et al., 2014; García-Alías et al., 2015) as well as based on its increased projections to the spinal cord (Bachmann et al., 2014; Zörner et al., 2014; García-Alías et al., 2015). In this context, a better knowledge of the properties of the corticobulbar projection in intact monkeys as reported here is welcome in order to assess to what extent it is modified following a lesion at various locations of the motor system (motor cortex, pyramidal tract, spinal cord, etc.) and how it may influence the reticulospinal system.

In conclusion, this study confirmed the hypothesis that the corticobulbar projections to PMRF in macaque monkeys differ with respect to the motor cortical area of origin in their density, mediolateral location of their terminal fields, rostro-caudal distribution along the PMRF, targeted nuclei of PMRF and laterality.

\section{Acknowledgements}

The authors thank Mrs Christine Roulin, Christiane Marti, Véronique Moret for their technical precious contributions to process the histological tissue and the animal care takers (L. Bossy, J. Maillard, B. Bapst, B. Morandi and J. Corpataux). Thanks are due to Dr. Michael Harvey for proofreading the English in the final version of the manuscript. This study was financially supported by Swiss National Science Foundation (SNF) grants to EMR, numbers $110005,132465,144990,149643$; grant Sinergia SNF number CRSII3_160696, and the Swiss Primate Competence Centre for Research (SPCCR: www.unifr.ch/specr)

\section{Conflict of interest}

The authors declare to have no conflict of interest in relation to this study.

\section{Author contributions}

EMR and $\mathrm{AH}$ designed the study and performed the tracers injections. MF, AC, $\mathrm{AH}$ and EMR analysed the histological sections. MF and EMR drafted the manuscript, reviewed by all authors.

\section{Data accessibility}

The histological material (sections) is publicly available at the SPCCR on request. The detailed reconstruction (equivalent of Fig. 3) of the corticobulbar projection in each monkey will be available in the Ph.D thesis manuscript of Michela Fregosi, available in the repository of Ph.D. theses completed at the Faculty of Sciences of University of Fribourg (http://opac.rero.ch/gateway?)

\section{Abbreviations}

BDA, biotinylated dextran amine; CB, Cholera toxin subunit B; CS, corticospinal; CST, Corticospinal tract; M1, Primary motor cortex; PMd, dorsal premotor cortex; PM, Premotor cortex; PMRF, Ponto-Medullary Reticular Formation; PMv, ventral premotor cortex; RST, Reticulopsinal tract; SMA, supplementary motor area. See Table 2 for abbreviations of brainstem nuclei.

\section{References}

Alstermark, B. \& Ekerot, C.F. (2015) The lateral reticular nucleus; integration of descending and ascending systems regulating voluntary forelimb movements. Front. Comput. Neurosci., 9, 102.

Alstermark, B. \& Ekerot, C.F. (2013) The lateral reticular nucleus: a precerebellar centre providing the cerebellum with overview and integration of motor functions at systems level. A new hypothesis. J. Physiol., 591, $5453-5458$.

Alstermark, B., Lindström, S., Lundberg, A. \& Sybirska, E. (1981) Integration in the descending motor pathways controlling the forelimb in the cat. 8. Ascending projection to the lateral reticular nucleus from $\mathrm{C} 3-\mathrm{C} 4$ propriospinal neurons also projecting to forelimb motoneurons. Exp. Brain Res., 42, 282-298.

Bachmann, L.C., Lindau, N.T., Felder, P. \& Schwab, M.E. (2014) Sprouting of brainstem-spinal tracts in response to unilateral motor cortex stroke in mice. J. Neurosci., 34, 3378-3389.

Baker, S.N., Zaaimi, B., Fisher, K.M., Edgley, S.A. \& Soteropoulos, D.S. (2015) Pathways mediating functional recovery. Prog. Brain Res., 218, 389-412.

Bashir, S., Kaeser, M., Wyss, A., Hamadjida, A., Liu, Y., Bloch, J., Brunet, J.F., Belhaj-Saif, A. et al. (2012) Short-term effects of unilateral lesion of the primary motor cortex (M1) on ipsilesional hand dexterity in adult macaque monkeys. Brain Struct. Funct., 217, 63-79.

Behzadi, G., Kalén, P., Parvopassu, F. \& Wiklund, L. (1990) Afferents to the median raphe nucleus of the rat: retrograde cholera toxin and wheat germ conjugated horseradish peroxidase tracing, and selective $\mathrm{D}-[3 \mathrm{H}]$ aspartate labelling of possible excitatory amino acid inputs. Neuroscience, 37, 77-100.

Benes, F.M. \& Lange, N. (2001) Two-dimensional versus three-dimensional cell counting: a practical perspective. Trends Neurosci., 24, 11-17.

Borra, E., Belmalih, A., Gerbella, M., Rozzi, S. \& Luppino, G. (2010) Projections of the hand field of the macaque ventral premotor area F5 to the brainstem and spinal cord. J. Comp. Neurol., 518, 2570-2591.

Buford, J.A. \& Davidson, A.G. (2004) Movement-related and preparatory activity in the reticulospinal system of the monkey. Exp. Brain Res., 159, 284-300.

Chouinard, P.A. \& Paus, T. (2006) The primary motor and premotor areas of the human cerebral cortex. Neuroscientist, 12, 143-152.

Courtine, G., Bunge, M.B., Fawcett, J.W., Grossman, R.G., Kaas, J.H., Lemon, R., Maier, I., Martin, J. et al. (2007) Can experiments in nonhuman primates expedite the translation of treatments for spinal cord injury in humans? Nat. Med., 13, 561-566.

Darling, W.G., Pizzimenti, M.A., Rotella, D.L., Peterson, C.R., Hynes, S.M., Ge, J., Solon, K., McNeal, D.W. et al. (2009) Volumetric effects of motor cortex injury on recovery of dexterous movements. Exp. Neurol., 220, 90108.

Darling, W.G., Morecraft, R.J., Rotella, D.L., Pizzimenti, M.A., Ge, J., Stilwell-Morecraft, K.S., Zhang, H., Soliman, H. et al. (2014) Recovery of precision grasping after motor cortex lesion does not require forced use of the impaired hand in macaca mulatta. Exp. Brain Res., 232, 3929-3938.

Davidson, A.G. \& Buford, J.A. (2004) Motor output from the primate reticular formation to shoulder muscles as revealed by stimulus-triggered averaging. J. Neurophysiol., 92, 83-95.

Davidson, A.G. \& Buford, J.A. (2006) Bilateral actions of the reticulospinal tract on arm and shoulder muscles in the monkey: stimulus triggered averaging. Exp. Brain Res., 173, 25-39.

Davidson, A.G., Schieber, M.H. \& Buford, J.A. (2007) Bilateral spike-triggered average effects in arm and shoulder muscles from the monkey pontomedullary reticular formation. J. Neurosci., 27, 8053-8058.

Drew, T., Dubuc, R. \& Rossignol, S. (1986) Discharge patterns of reticulospinal and other reticular neurons in chronic, unrestrained cats walking on a treadmill. J. Neurophysiol., 55, 375-401.

Dum, R.P. \& Strick, P.L. (1991) The origin of corticospinal projections from the premotor areas in the frontal lobe. J. Neurosci., 11, 667-689.

Dum, R.P. \& Strick, P.L. (1996) Spinal cord terminations of the medial wall motor areas in macaque monkeys. J. Neurosci., 16, 6513-6525.

Dum, R.P. \& Strick, P.L. (2002) Motor areas in the frontal lobe of the primate. Physiol. Behav., 77, 677-682.

Esposito, M.S., Capelli, P. \& Arber, S. (2014) Brainstem nucleus MdV mediates skilled forelimb motor tasks. Nature, 508, 351-356. 
García-Alías, G., Truong, K., Shah, P.K., Roy, R.R. \& Edgerton, V.R. (2015) Plasticity of subcortical pathways promote recovery of skilled hand function in rats after corticospinal and rubrospinal tract injuries. Exp. Neurol., 266, 112-119.

Geuna, S. (2000) Appreciating the difference between design-based and model-based sampling strategies in quantitative morphology of the nervous system. J Comp Neurol, 427, 333-339.

Hamadjida, A., Wyss, A.F., Mir, A., Schwab, M.E., Belhaj-Saif, A. \& Rouiller, E.M. (2012) Influence of anti-Nogo-A antibody treatment on the reorganization of callosal connectivity of the premotor cortical areas following unilateral lesion of primary motor cortex (M1) in adult macaque monkeys. Exp. Brain Res., 223, 321-340.

He, S.Q., Dum, R.P. \& Strick, P.L. (1993) Topographic organization of corticospinal projections from the frontal lobe: motor areas on the lateral surface of the hemisphere. J. Neurosci., 13, 952-980.

He, S.Q., Dum, R.P. \& Strick, P.L. (1995) Topographic organization of corticospinal projections from the frontal lobe: motor areas on the medial surface of the hemisphere. J. Neurosci., 15, 3284-3306.

Honeycutt, C.F., Kharouta, M. \& Perreault, E.J. (2013) Evidence for reticulospinal contributions to coordinated finger movements in humans. J. Neurophysiol., 110, 1476-1483.

Hoogewoud, F., Hamadjida, A., Wyss, A.F., Mir, A., Schwab, M.E., BelhajSaif, A. \& Rouiller, E.M. (2013) Comparison of functional recovery of manual dexterity after unilateral spinal cord lesion or motor cortex lesion in adult macaque monkeys. Front Neurol., 4, 101.

Hornung, J.P. (2003) The human raphe nuclei and the serotonergic system. J. Chem. Neuroanat., 26, 331-343.

Isa, T., Kinoshita, M. \& Nishimura, Y. (2013) Role of direct vs. indirect pathways from the motor cortex to spinal motoneurons in the control of hand dexterity. Front Neurol., 4, 191

Jacobs, B.L. \& Azmitia, E. (1992) Structure and function of the brain serotonin system. Physiol. Rev., 72, 165-229.

Jenny, A.B. \& Inukai, J. (1983) Principles of motor organization of the monkey cervical spinal cord. J. Neurosci., 3, 567-575.

Kaeser, M., Wyss, A.F., Bashir, S., Hamadjida, A., Liu, Y., Bloch, J., Brunet, J.F., Belhaj-Saif, A. et al. (2010) Effects of unilateral motor cortex lesion on ipsilesional hand's reach and grasp performance in monkeys: relationship with recovery in the contralesional hand. J. Neurophysiol., 103, 1630-1645.

Kaeser, M., Brunet, J.F., Wyss, A., Belhaj-Saif, A., Liu, Y., Hamadjida, A., Rouiller, E.M. \& Bloch, J. (2011) Autologous adult cortical cell transplantation enhances functional recovery following unilateral lesion of motor cortex in primates: a pilot study. Neurosurgery, 68, 1405-1416.

Kazennikov, O., Hyland, B., Corboz, M., Babalian, A., Rouiller, E.M. \& Wiesendanger, M. (1999) Neural activity of supplementary and primary motor areas in monkeys and its relation to bimanual and unimanual movement sequences. Neuroscience, 89, 661-674.

Keizer, K. \& Kuypers, H.G.J.M. (1989) Distribution of corticospinal neurons with collaterals to the lower brain stem reticular formation in monkey (Macaca fascicularis). Exp. Brain Res., 7, 311-318.

Kermadi, I., Liu, Y., Tempini, A., Calciati, E. \& Rouiller, E.M. (1998) Neuronal activity in the primate supplementary motor area and the primary motor cortex in relation to spatio-temporal bimanual coordination. Somatosens. Mot. Res., 15, 287-308

Kermadi, I., Liu, Y. \& Rouiller, E.M. (2000) Do bimanual motor actions involve the dorsal premotor (PMd), cingulate (CMA) and posterior parietal (PPC) cortices ? Comparison with primary and supplementary motor cortical areas. Somatosens. Mot. Res., 17, 255-271.

Kuypers, H.G.J.M. (1958) Some projections from the peri-central cortex to the pons and lower brain stem in monkey and chimpanzee. J. Comp. Neurol., 110, 221-255.

Kuypers, H.G.J.M. (1981). Anatomy of the descending pathways. In Handbook of Physiology. The Nervous System II. American Physiol Society, Bethesda, pp. 597-666.

Kuypers, H.G.J.M. \& Lawrence, D.G. (1967) Cortical projections to the red nucleus and the brainstem in the rhesus monkey. Brain Res., 4, 151-188.

Lavenex, P., Steele, M.A. \& Jacobs, L.F. (2000) The seasonal pattern of cell proliferation and neuron number in the dentate gyrus of wild adult eastern grey squirrels. Eur. J. Neurosci., 12, 643-648.

Lawrence, D.G. \& Kuypers, H.G.J.M. (1968a) The functional organization of the motor system in the monkey. I. The effects of bilateral pyramidal lesions. Brain, 91, 1-14.

Lawrence, D.G. \& Kuypers, H.G.J.M. (1968b) The functional organization of the motor system in the monkey. II. The effects of lesions of the descending brain-stem pathways. Brain, 91, 15-36.
Lemon, R.N. (1993) The cortical control of the primate hand. G.L. Brown Prize Lecture. Exp. Physiol., 78, 263-301.

Lemon, R.N. (2008) Descending pathways in motor control. Annu. Rev. Neurosci., 31, 195-218.

Lemon, R. N. (2010) What drives corticospinal output? F1000 Biol. Rep., 2,

Lemon, R.N. (2016) Cortical projections to the red nucleus and the brainstem in the rhesus monkey. Brain Res., 1645, 28-30.

Lemon, R.N. \& Griffiths, J. (2005) Comparing the function of the corticospinal system in different species: organizational differences for motor specialization. Muscle Nerve, 32, 261-279.

Lemon, R.N., Landau, W., Tutssel, D. \& Lawrence, D.G. (2012) Lawrence and Kuypers (1968a, b) revisited: copies of the original filmed material from their classic papers in Brain. Brain, 135, 2290-2295.

Liang, H., Watson, C. \& Paxinos, G. (2015) Projections from the oral pontine reticular nucleus to the spinal cord of the mouse. Neurosci. Lett., 584, $113-118$

Liang, H., Watson, C. \& Paxinos, G. (2016) Terminations of reticulospinal fibers originating from the gigantocellular reticular formation in the mouse spinal cord. Brain Struct. Funct., 221, 1623-1633.

Liu, Y. \& Rouiller, E.M. (1999) Mechanisms of recovery of dexterity following unilateral lesion of the sensorimotor cortex in adult monkeys. Exp. Brain Res., 128, 149-159.

Marini, G. \& Wiesendanger, M. (1987) Cortical and peripheral effects on single neurons of the lateral reticular nucleus in the monkey. J. Comp Neurol., 256, 581-589.

Matsuyama, K. \& Drew, T. (1997) Organization of the projections from the pericruciate cortex to the pontomedullary brainstem of the cat: a study using the anterogrande tracer Phaseolus vulgaris-Leucoagglutinin. J. Comp Neurol., 389, 617-641.

Matsuyama, K., Takakusaki, K., Nakajima, K. \& Mori, S. (1997) Multi-segmental innervation of single pontine reticulospinal axons in the cervicothoracic region of the cat: anterograde PHA-L tracing study. J. Comp Neurol., 377, 234-250.

Matsuyama, K., Mori, F., Kuze, B. \& Mori, S. (1999) Morphology of single pontine reticulospinal axons in the lumbar enlargement of the cat: a study using the anterograde tracer PHA-L. J. Comp Neurol., 410, 413-430.

Matsuyama, K., Mori, F., Nakajima, K., Drew, T., Aoki, M. \& Mori, S. (2004) Locomotor role of the corticoreticular-reticulospinal-spinal interneuronal system. Prog Brain Res., 143, 239-249.

Murray, E.A. \& Coulter, J.D. (1981) Organization of corticospinal neurons in the monkey. J. Comp Neurol., 195, 339-365.

Myers, B., Scheimann, J.R., Franco-Villanueva, A. \& Herman, J.P. (2016) Ascending mechanisms of stress integration: Implications for brainstem regulation of neuroendocrine and behavioral stress responses. Neurosci. Biobehav. R., 74, 366-375.

Ortiz-Rosario, A., Berrios-Torres, I., Adeli, H. \& Buford, J.A. (2014) Combined corticospinal and reticulospinal effects on upper limb muscles. $\mathrm{Neu}$ rosci. Lett., 561, 30-34.

Paxinos, G., Huang, X.F. \& Toga, A.W. (2000) The Rhesus Monkey Brain in Stereotaxic Coordinates. Academic Press, San-Diego, London. ISBN: $0-12-358255-5$

Perrier, J.F. \& Cotel, F. (2015) Serotonergic modulation of spinal motor control. Curr. Opin. Neurobiol., 33, 1-7.

Pizzimenti, M.A., Darling, W.G., Rotella, D.L., McNeal, D.W., Herrick, J.L., Ge, J., Stilwell-Morecraft, K.S. \& Morecraft, R.J. (2007) Measurement of reaching kinematics and prehensile dexterity in nonhuman primates. $J$. Neurophysiol., 98, 1015-1029.

Rathelot, J.A. \& Strick, P.L. (2009) Subdivisions of primary motor cortex based on cortico-motoneuronal cells. Proc. Natl. Acad. Sci. USA, 106, 918-923

Rho, M.J., Cabana, T. \& Drew, T. (1997) Organization of the projections from the pericruciate cortex to the pontomedullary reticular formation of the cat: a quantitative retrograde tracing study. J. Comp Neurol., 388, 228-249

Riddle, C.N. \& Baker, S.N. (2010) Convergence of pyramidal and medial brain stem descending pathways onto macaque cervical spinal interneurons. J. Neurophysiol., 103, 2821-2832.

Riddle, C.N., Edgley, S.A. \& Baker, S.N. (2009) Direct and indirect connections with upper limb motoneurons from the primate reticulospinal tract. $J$. Neurosci., 29, 4993-4999.

Rouiller, E.M., Babalian, A.-, Kazennikov, O., Moret, V., Yu, X.H. \& Wiesendanger, M. (1994) Transcallosal connections of the distal forelimb representations of the primary and supplementary motor cortical areas in macaque monkeys. Exp. Brain Res., 102, 227-243. 
Rouiller, E.M., Moret, V., Tanne, J. \& Boussaoud, D. (1996) Evidence for direct connections between the hand region of the supplementary motor area and cervical motoneurons in the macaque monkey. Eur. J. Neurosci., 8, 1055-1059.

Sakai, S.T., Davidson, A.G. \& Buford, J.A. (2009) Reticulospinal neurons in the pontomedullary reticular formation of the monkey (Macaca fascicularis). Neuroscience, 163, 1158-1170.

Schepens, B. \& Drew, T. (2004) Independent and convergent signals from the pontomedullary reticular formation contribute to the control of posture and movement during reaching in the cat. J. Neurophysiol., 92, 2217-2238.

Schepens, B. \& Drew, T. (2006) Descending signals from the pontomedullary reticular formation are bilateral, asymmetric, and gated during reaching movements in the cat. J. Neurophysiol., 96, 2229-2252.

Schepens, B., Stapley, P. \& Drew, T. (2008) Neurons in the pontomedullary reticular formation signal posture and movement both as an integrated behavior and independently. J. Neurophysiol., 100, 2235-2253.

Schieber, M.H. (2007) Motor neuron disorders and related diseases. Chapter2 Comparative anatomy and physiology of the corticospinal system. Handb. Clin. Neurol., 82, 15-37.

Soteropoulos, D.S., Williams, E.R. \& Baker, S.N. (2012) Cells on the monkey ponto-medullary reticular formation modulate their activity with slow finger movements. J. Physiol., 590, 4011-4027.

Valverde, F. (1961) Reticular formation of the pons and medulla oblongata. A Golgi study. J. Comp Neurol., 116, 71-99.
Valverde, F. (1962) Reticular formation of the albino rat's brain stem cytoarchitecture and corticofugal connections. J. Comp Neurol., 119, 25-53.

Wiesendanger, R. \& Wiesendanger, M. (1987) Topography of the corticofugal projection to the lateral reticular nucleus in the monkey. J. Comp Neurol., 256, 570-580.

Wiesendanger, R., Wiesendanger, M. \& Rüegg, D.G. (1979) An anatomical investigation of the corticopontine projection in the primate (Macaca fascicularis and Saimiri sciureus)-II. The projection from frontal and parietal association areas. Neuroscience, 4, 747-765.

Wiesendanger, M., Rouiller, E.M., Kazennikov, O. \& Perrig, S. (1996) Is the supplementary motor area a bilaterally organized system? In: Lüders, H.O. (Ed), Advances in Neurology, Vol. 70: Supplementary Motor Area. Lippincott-Raven Publishers, Philadelphia, pp. 85-93.

Wyss, A.F., Hamadjida, A., Savidan, J., Liu, Y., Bashir, S., Mir, A., Schwab, M.E., Rouiller, E.M. et al. (2013) Long-term motor cortical map changes following unilateral lesion of the hand representation in the motor cortex in macaque monkeys showing functional recovery of hand functions. Restor. Neurol. Neuros., 31, 733-760.

Zaaimi, B., Edgley, S.A., Soteropoulos, D.S. \& Baker, S.N. (2012) Changes in descending motor pathway connectivity after corticospinal tract lesion in macaque monkey. Brain, 135, 2277-2289.

Zörner, B., Bachmann, L.C., Filli, L., Kapitza, S., Gullo, M., Bolliger, M. Starkey, M.L., Röthlisberger, M. et al. (2014) Chasing central nervous system plasticity: the brainstem's contribution to locomotor recovery in rats with spinal cord injury. Brain, 137, 1716-1732. 